\title{
Algebra for Databases with Explicit Markings of Damaged Data
}

Giovanni Rumolo

Dip. di Informatica ed Automazione, Università di Roma TRE

Via della Vasca Navale, 79 - 00146 ROMA Italy. rumolo@dia.uniroma3.it

Sushil Jajodia

The MITRE Corporation

1820 Dolley Madison Boulevard, McLean, VA 22102, USA. jajodia@mitre.org

\begin{abstract}
This paper deals with the management of data with different integrity grades, represented by marked data. In databases with explicit markings of damaged data, which integrity constraints apply depends on the markings of the referenced data. Correct data conform to the full set of integrity constraints while for data that is either damaged, but inessential or approximate, some integrity constraints can be relaxed. The main goal of this paper is to provide a foundation for marked databases extending the relational algebra. Results provided in this paper are preliminary, but they provide a pragmatic and reasonable approach without sacrificing the theoretical foundation.
\end{abstract}

\section{INTRODUCTION.}

In databases, integrity is traditionally viewed as Boolean: a database either has integrity or it does not. A database that satisfies formally specified integrity constraints nonetheless diverges, sometimes seriously, from the real world. Many factors inhibit synchronization with the external world. Aside from the initial modeling problem, both the real world and the system evolve over time. Experience has shown that keeping up with the evolution is timeconsuming and error-prone. Even worse, some systems evolve due to malicious activity. A malicious user, an offender, may install incorrect information in the database either for personal benefit, such as fraudulently acquiring goods or services, or with the intent of damaging the organization's operation and fulfillment of its mission through disruption of its information systems [1].

Researchers have been investigating ways to address the incompleteness and inconsistency that occur in real systems. For example, relaxing integrity constraints improves concurrent access in distributed databases $[2,17,10]$; ex- 
plicit management of inconsistent value extends the range of usable values for user applications [16, 21]; extending semantics of null values improves system flexibility [5]; explicit management of indefinite and incomplete information gives rise to indefinite as well as maybe query answers [7, 18].

This paper deals with the management of different integrity grades represented by marked data. We consider the scheme proposed by Ammann et al. [1] to maintain precise information about the integrity of data using different color markers. They provide sophisticated algorithms to identify integrity deviations, mark inconsistent data, track and contain the spread of inconsistency, manage repair, and oversee a return to normal service.

The main goal of this work is to provide a foundation for marked databases that is able to capture specific features of marked data and to extend the relational algebra in a coherent way.

The work is presented as follows: Section 2 introduces informally the marked data and gives some preliminary examples; Section 3 introduces rigorously marked databases; Section 4 defines formally the logic framework for marked databases while Section 5 provides the relational algebra for marked data and some of its properties; Section 6 discusses related works; and Section 7 presents conclusions.

\section{MARKED DATA OVERVIEW}

In a database with explicit markings of damaged data, each marker represents a different grade of data reliability for user applications. As presented in [1], we introduce four different markers and associate them with different colors:

Red, $[\square \mathbf{R}]$ : damaged data that are always not applicable; they are provided by the offender and they are essential to a correct system behavior. Red data are similar to null values that violate database integrity constraints;

Off-Red. $[\circ \mathbf{R}]$ : damaged data that are sometimes not applicable; they are provided by the offender and they are not essential. Off-Red data are similar to null values that do not violate database integrity constraints;

Off-Green, $[\circ \mathrm{G}]$ : damaged data that are sometimes applicable; they are provided by the system administrator (the defender) and they are in some sense equivalent to the original data. OFF-Green data result in the correct system behavior and they can be back-up values that do not violate the database integrity constraints;

Green, $[\square \mathbf{G}]$ : undamaged data that are always applicable; specified values for these data always satisfy the database integrity constraints.

There are two frameworks that are relevant to our problem: modal logic of necessity and possibility $[11,15,20]$ and database theory of null values $[5,8$, 12]. In this work we attempt, using modal logic, to formalize the main feature 
of marked data, that is: marked data are data that someone wants or must use.

We employ the following example to illustrate our ideas. Suppose that in a department of some organization it is necessary to store information about telephone lines. Department managers want to know in which room each telephone lies and which person can answer a telephone call. Moreover, they want know who is responsible for the payment of each telephone bill. The relations in Tables 1 and 2 give the database state, where next to each values there is a color marker.

\begin{tabular}{|c|c|c|}
\hline \multicolumn{3}{|c|}{ PERSON } \\
\hline NAME & SURNAME & SSN \\
\hline John:Q G & Freeman:DG & 234599133:० R \\
\hline Paul:॰G & White:0 G & $324499581: 0 \mathrm{G}$ \\
\hline Kathy: $\bullet \mathbf{G}$ & Taylor: $\bullet \mathbf{G}$ & $325599585: \circ \mathbf{G}$ \\
\hline Lucy: $\mathbf{G}$ & Wolf: $\bullet \mathbf{G}$ & $324499581: 0 \mathbf{R}$ \\
\hline Sam: : R & Huck: $0 \mathbf{R}$ & 245569789:0G \\
\hline
\end{tabular}

TELEPHONE

\begin{tabular}{ll}
\hline TELEPHONE & COMPANY \\
\hline 703-708-4427:० G & AT\&T:०R \\
\hline 703-708-4429:०R & AT\&T:OG \\
\hline 703-993-1629:0 G & Sprint:0 G \\
\hline 703-993-1628:0 R & Sprint:0 R \\
\hline
\end{tabular}

Table 1 Marked Tables person and telephone

\begin{tabular}{|c|c|c|}
\hline SSN & ROOM & TELEPHONE \\
\hline 234599133:口G & $301: \square G$ & 703-993-1629:口G \\
\hline $324599587: 0 G$ & 301:०G & 703-708-4429:口G \\
\hline $325599585: 0 G$ & $211: 0 G$ & 703-993-1628:口 R \\
\hline 324499581:० R & 211:0G & $703-708-4427: 0 \mathrm{G}$ \\
\hline 245569789:०G & $302: \circ \mathbf{G}$ & 703-993-1629:० R \\
\hline 245569789:०R & 212:0 R & 703-708-4429:0 R \\
\hline 245569787:0 R & $303: 0 \mathrm{G}$ & 703-993-1628:० $\mathbf{R}$ \\
\hline
\end{tabular}

PAYMENT

\begin{tabular}{cc}
\hline SSN & TELEPHONE \\
\hline $234599133: 0$ R & $703-708-4429: 0$ G \\
\hline $234599133: 0$ G & $703-708-4428: 0$ R \\
\hline 234599133:0 R & $703-993-1629: 0$ G \\
\hline $245569789: 0$ G & $703-993-1628: 0$ G \\
\hline
\end{tabular}

Table 2 Marked Tables person-room-telephone and PAY-For

Suppose we want the list of all known persons in relation PERson. If we consider all attributes in a relation when we display the row $\langle$ Lucy: $\square \mathbf{G}$, Wolf : $\diamond \mathrm{G}, 324499581: \square \mathbf{R}>$ the user will be informed by $\square \mathbf{R}$ marker next to SSN code that it is not valid. That is, the value should not be used and it should not be in the query result. Instead, if the user were interested in the first two columns, he or she could use the data. Consider the case of selecting all tuples from relational table PERSON-ROOM-TELEPHONE where " ROOM > 199 and ROOM < 300"'. The evaluation of the condition depends on both markers and values stored in the relational table. Again, all Red values are not valid; 
Off-Red values must be used even though they are damaged. Consider the case of making the union between relational table PAYMENT and the projection of first and last column of relational table PERSON-ROOM-TELEPHONE. Many tuples identical in value but different for the markers can occur but users would like to see just one tuple, the most useful.

Markers introduce many problems in behavior of databases. Not for all situations is it possible to accomplish automatic management of marked data, but the user must be considered. A database system for marked data should provide standard models of behavior leaving users free to choose one of them. Each model of behavior should define increasing visibility over damaged data.

\section{FOUNDATION FOR MARKED DATABASES}

We define a relational algebra over marked data giving a value-based [3] semantics for marked databases. We use the standard definition for relational databases distinguishing marked objects with a superscript ${ }^{c}$. In a marked tuple $t^{c}$ we consider values explicitly marked with a marker $C \in\{\square \mathbf{G}, \diamond \mathbf{G}$, $\diamond \mathbf{R}, \square \mathbf{R}\}$. Let us denote, for a given database scheme $D B=\left\{R_{1}\left(A_{1}, A_{2}\right.\right.$, $\left.\left.\ldots, A_{n}\right), R_{2}\left(B_{1}, B_{2}, \ldots, B_{m}\right), \ldots, R_{k}\left(C_{1}, C_{2}, \ldots, C_{l}\right)\right\}$, with $\mathcal{U}^{c}$ the set of all marked relations over every finite subset of attributes $U_{D B}$, with $\mathcal{U}$ the set of all corresponding relations for the same database scheme and with $\mathcal{I}(D B)^{c}$, $\mathcal{I}(D B)$ database instances. For each attribute $A_{j}$ we denote with $\operatorname{dom}^{c}\left(A_{j}\right)$ its marked domain and with $\operatorname{dom}\left(A_{j}\right)$ its corresponding domain. Moreover, for a scheme $X\left(A_{1}, A_{2}, \ldots, A_{k}\right)$ we denote with $\operatorname{dom}^{c}(X)=\operatorname{dom}^{c}\left(A_{1}\right) \times$ $\operatorname{dom}^{c}\left(A_{2}\right) \times \ldots \times \operatorname{dom}^{c}\left(A_{k}\right)$ the domain of the scheme. We assume that a set of projection functions $\Pi=\left\{\pi_{A_{j}}: \operatorname{dom}(X) \rightarrow \operatorname{dom}\left(A_{j}\right) \mid\right.$ for each $\left.A_{j} \in X\right\}$ are defined. We formalize the meaning of each marker with respect to the set of all possible marked queries and marked domains. We consider only computable queries as defined by Chandra [6].

Definition 1 (CH-computable) A computable query is a partial recursive function which, given a database as input, produces as output a relation on the domain of the database, and satisfies a consistency criterion.

We assign to a marked query $\mathcal{Q}^{c}$ the type: $\mathcal{Q}^{c}: \mathcal{I}(D B)^{c} \rightarrow \mathcal{U}^{c}$. Let us denote with $\mho_{\mathcal{I}(D B)^{c}}=\mathcal{U}^{c^{\mathcal{I}(D B)^{c}}}$ the space of all $\mathcal{Q}^{c}$ on $\mathcal{I}(D B)^{c}$ and with $\mathcal{Q}^{c}\left(\boldsymbol{r}^{c}\right)$ the result of $\mathcal{Q}^{c}$ on $\boldsymbol{r}^{c}$.

Queries are expressed by ordinary relational algebra expressions and for each $\mathcal{Q}$ (marked or not) there exists, at least, one expression $\mathcal{E}$ that represents the query and such that $\mathcal{E}(\boldsymbol{r})=\mathcal{Q}(\boldsymbol{r})$. Let us denote with $\mathcal{S}(\mathcal{Q})=X_{1}, X_{2}, \ldots$, $X_{k}$ the scheme for $\mathcal{E}(\boldsymbol{r})=\mathcal{Q}(\boldsymbol{r})$. The codomain $\operatorname{im}(\mathcal{Q})$ of $\mathcal{Q}(\boldsymbol{r})$ is a subset of $\operatorname{dom}(X)$ and we denote it $\operatorname{dom}(\mathcal{S}(\mathcal{Q}))$. 
Because a marked database should behave, as much as possible, as an ordinary database we do not consider in the syntax of querying the markers.

\section{THE MODAL LOGIC OF COLORS}

We give the formalization of color markers using a propositional modal logic of colors. In Appendix 1 we introduce standard syntax and semantics of propositional modal logic $\mathcal{L}_{\mathcal{M L}}$ following [11]. Moreover, in Section 4.2 we introduce a mapping between $\mathcal{L}_{\mathcal{M L}}$ formulas provided for marked databases and a standard three-valued propositional logic $\mathcal{L}_{\mathcal{M} \mathcal{V}^{3}}[20] . \mathcal{L}_{\mathcal{M L}}$ and $\mathcal{L}_{\mathcal{M} \mathcal{V}^{3}}$ characterize relevant properties of the relational algebra for marked relations.

We define semantics of color markers relating the membership of a value in a marked domain with the result of a marked query. Using modal logics we code each marked value as a quasi-atomic formula and each marked tuple as a conjunction of quasi-atomic formulas (see Appendix 1).

Definition 2 The meaning of each $C \in\{\square \mathbf{G}, \diamond \mathbf{G}, \diamond \mathbf{R}, \square \mathbf{R}\}$, for $t^{c}\left[A_{j}\right] \equiv$ $t\left[A_{j}\right]: C_{j}$ of a tuple $t^{c} \in r^{c} i s^{*}$ :

$$
\begin{aligned}
t\left[A_{j}\right]: \square \mathbf{G}_{j} & \equiv \forall \mathcal{Q}^{c} \in \mho_{\mathcal{I}(D B)^{c}}\left(\left(A_{j} \in \mathcal{S}\left(\mathcal{Q}^{c}\right)\right) \rightarrow t^{c}\left[A_{j}\right] \in \operatorname{dom}^{c}\left(\pi_{A_{j}}\left(\mathcal{S}\left(\mathcal{Q}^{c}\right)\right)\right)\right)(1) \\
t\left[A_{j}\right]: \square \mathbf{R}_{j} & \equiv \forall \mathcal{Q}^{c} \in \mho_{\mathcal{I}(D B)^{c}}\left(\left(A_{j} \in \mathcal{S}\left(\mathcal{Q}^{c}\right)\right) \rightarrow t^{c}\left[A_{j}\right] \notin \operatorname{dom}^{c}\left(\pi_{A_{j}}\left(\mathcal{S}\left(\mathcal{Q}^{c}\right)\right)\right)\right)(2) \\
t\left[A_{j}\right]: \diamond \mathbf{G}_{j} & \equiv \exists \mathcal{Q}^{c} \in \mho_{\mathcal{I}(D B)^{c}}\left(\left(A_{j} \in \mathcal{S}\left(\mathcal{Q}^{c}\right)\right) \wedge t^{c}\left[A_{j}\right] \in \operatorname{dom}^{c}\left(\pi_{A_{j}}\left(\mathcal{S}\left(\mathcal{Q}^{c}\right)\right)\right)\right) \\
t\left[A_{j}\right]: \diamond \mathbf{R}_{j} & \equiv \exists \mathcal{Q}^{c} \in \mho_{\mathcal{I}(D B)^{c}}\left(\left(A_{j} \in \mathcal{S}\left(\mathcal{Q}^{c}\right)\right) \wedge t^{c}\left[A_{j}\right] \notin \operatorname{dom}^{c}\left(\pi_{A_{j}}\left(\mathcal{S}\left(\mathcal{Q}^{c}\right)\right)\right)\right)
\end{aligned}
$$

We consider each of the above definitions a value constraint where: $A_{j} \in$ $\mathcal{S}\left(\mathcal{Q}^{c}\right)$ asserts that values in $\operatorname{dom}^{c}\left(A_{j}\right)$ are accessible through $\mathcal{S}\left(\mathcal{Q}^{c}\right) ; t^{c}\left[A_{j}\right](\notin$ )$\in \operatorname{dom}^{c}\left(\pi_{A_{j}}\left(\mathcal{S}\left(\mathcal{Q}^{c}\right)\right)\right)$ asserts that $t^{c}\left[A_{j}\right] \equiv t\left[A_{j}\right]: C_{j}$ belongs (or not) to $\operatorname{dom}^{c}\left(A_{j}\right) . \pi_{A_{j}}$ links $t^{c}\left[A_{j}\right]$ with the exact image of $\mathcal{Q}^{c}$. Each value constraint is a necessary condition that asserts when the marked value should be in the domain of the proper attribute of the query result.

Definition 3 (Tuple constraint in $\mathcal{L}_{\mathcal{M L}}$ ) Given $t^{c} \in \boldsymbol{r}^{c}$ defined on $X\left(A_{1}\right.$, $\left.A_{2}, \ldots, A_{k}\right)$, with $t^{c}=<t^{c}\left[A_{1}\right], t^{c}\left[A_{2}\right], \ldots, t^{c}\left[A_{k}\right]>$, where for $t^{c}\left[A_{j}\right] \equiv t\left[A_{j}\right]:$ $C_{j}$ is $t\left[A_{j}\right] \in \operatorname{dom}\left(A_{j}\right)$ and $C_{j} \in\{\square \mathbf{G}, \diamond \mathbf{G}, \diamond \mathbf{R}, \square \mathbf{R}\}$, the tuple constraint for $t^{c}$ is in $\mathcal{L}_{\mathcal{M L}}$ the following conjunction of quasi atomic formulas:

$$
\begin{aligned}
& \psi^{c}\left(X, t^{c}\right) \equiv \bigwedge_{j=1 \ldots k} \phi^{c}\left(t\left[A_{j}\right]: C_{j}\right) \\
& \phi^{c}\left(t\left[A_{j}\right]: \square \mathbf{G}\right) \equiv \square t\left[A_{j}\right] \quad \phi^{c}\left(t\left[A_{j}\right]: \diamond \mathbf{G}\right) \equiv \diamond t\left[A_{j}\right] \\
& \phi^{c}\left(t\left[A_{j}\right]: \square \mathbf{R}\right) \equiv \square \neg t\left[A_{j}\right] \quad \phi^{c}\left(t\left[A_{j}\right]: \diamond \mathbf{R}\right) \equiv \diamond \neg t\left[A_{j}\right]
\end{aligned}
$$

*Here, logic connectives are used as shortcuts of the following words: $\{\forall \equiv$ "for every", $\exists \equiv$ "exists",$\wedge \equiv$ "and", $\vee \equiv$ "or", $\rightarrow \equiv$ "implies"\} 
$\mathcal{I}(D B)^{c}$ defines a set $D B=\left\{\psi^{c}\left(X, t^{c}\right) \mid \forall t^{c} \in \mathcal{I}(D B)^{c}, t^{c}\right.$ defined on $\left.X\right\}$ of tuple constraints.

We consider those models $\mathcal{M}_{D B}=\left(\mathcal{W}_{D B}, \mathcal{R}_{D B}, \mathcal{V}_{D B}\right)$ for the set $D B$ of tuple constraints where each set $w \subseteq 2^{\mathcal{U}^{c}}$ of marked relations over every finite subset of attributes $U_{D B}$ is a possible world $w \in \mathcal{W}_{D B}$, the query space $\mho_{\mathcal{I}(D B)^{c}}$ defines the accessibility relations $\mathcal{R}_{D B}$ between possible worlds and the assignment $\mathcal{V}_{D B}: \mathcal{P}_{D B} \mapsto 2^{\mathcal{W}_{D B}}$ defines in which worlds a value belongs to the result of a query. Under these models, Equation 1 means that green data are those that belongs to every accessible world; that is, they should be necessarily true in all accessible worlds. Equation 2 says that red data cannot belong to the result of a marked query, even if they are in the database, that is they should be necessarily false in all accessible worlds. For Off-colors the given semantics mean that "the marked value belongs to the result only for some of those marked queries where it should appear". Equation 3 means "it is possible that it does appear" and Equation 4 means "it is possible that it does not appear".

\subsection{Coding Marked Data into S5}

Now we show some property of the coding of $\mathcal{I}(D B)^{c}$ into the modal logic S5.

Definition 4 (Color consistency) $\mathcal{I}(D B)^{c}$ is color consistent iff $D B \nvdash_{S 5} \perp$

Definition 5 (Color satisfiability) $\mathcal{I}(D B)^{c}$ is color satisfiable iff $\exists \mathcal{M}_{S 5}, \mathcal{M}_{S 5} \models_{S 5} D B$

Proposition 1 (Color inconsistency) $A$ set $D B$ of tuple constraints is consistent in $S 5$ (and more in general in KD) iff for any propositional variable $t\left[A_{j}\right] \in \mathcal{P}$ does not occur for some $\psi^{c}\left(X, t^{c}\right)$ one of the following three cases:
(a) $\square \mathbf{G} t\left[A_{j}\right] \wedge \diamond \mathbf{R} t\left[A_{j}\right]$
(b) $\diamond \mathbf{G} t\left[A_{j}\right] \wedge \square \mathbf{R} t\left[A_{j}\right]$
(c) $\square \mathbf{G} t\left[A_{j}\right] \wedge \square \mathbf{R} t\left[A_{j}\right]$

Proposition 2 (Color unsatisfiability) $A$ color consistent set $D B$ of tuple constraints is satisfiable in $S 5$ iff any propositional variable $t\left[A_{j}\right] \in \mathcal{P}$ does not occur in $D B$ in one of the following conflicting forms:
(a) $\square \mathbf{G t}\left[A_{j}\right]$ and $\diamond \mathbf{R} t\left[A_{j}\right]$
(b) $\diamond \mathbf{G} t\left[A_{j}\right]$ and $\square \mathbf{R} t\left[A_{j}\right]$
(c) $\square \mathbf{G t}\left[A_{j}\right]$ and $\square \mathbf{R} t\left[A_{j}\right]$

Color consistency is a necessary condition to grant that any query cannot draw out contradictory information from a single tuple. Color satisfiability is a necessary and sufficient condition to grant that any query cannot draw out contradictory information from all values in a marked database. 
Proposition 3 (Model existence) The model $\mathcal{M}^{*}=\left(\mathcal{F}^{*}, \mathcal{V}^{*}\right)$ where $\mathcal{F}^{*}$ is shown in Figure 1 and $\mathcal{V}^{*}$ is an $S 5$ model for any color satisfiable set $D B$ of tuple constraints:

$$
\mathcal{V}^{*}\left(t\left[A_{j}\right]\right)= \begin{cases}\{T, \perp\} & \text { if } \square t\left[A_{j}\right] \text { occurs in } D B \\ \{T\} & \text { if } \diamond t\left[A_{j}\right] \text { occurs in } D B\end{cases}
$$

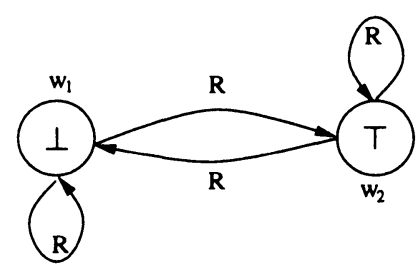

Figure 1 The Frame $\mathcal{F}^{*}$

It is not necessary to use the standard proof theory of S5 to verify color satisfiability and the problem is solvable in at most polynomial time bounded by the number of different values in the active domain of a marked database. In fact (see [22]), for $\mathcal{L}_{\mathcal{M} \mathcal{L}}$ the following holds:

Proposition 4 There is an algorithm that, given a finite Kripke model $\mathcal{M}=$ $(\mathcal{W}, \mathcal{R}, \mathcal{V})$, a world $w \in \mathcal{W}$ and a formula $\mathcal{F} \in \mathcal{L}_{\mathcal{M L}}$, determines whether $\mathcal{M} \models_{w} \mathcal{F}$ in time $O(|\mathcal{M}| \times|\mathcal{F}|)$.

Although we consider the modal logic S5 as the right framework where we can develop a complete theory of marked databases, including marked integrity constraints and functional dependencies, we use a mapping of S5 into a propositional three-valued logic to show, in an effective way, how we verify query consistency and how we evaluate marked queries.

\subsection{Coding Formulas over Marked Data into $\mathcal{L}_{\mathcal{M} \mathcal{V}^{3}}$}

We use the propositional three-valued logic $\mathcal{L}_{\mathcal{M} \nu^{3}}$ proposed by Lukasiewics [20] to define an effective interpretation of logical expressions over marked values. Because $\mathcal{L}_{\mathcal{M} \mathcal{V}^{3}}$ is a particular case of propositional multi-valued logic $\mathcal{L}_{\mathcal{M V}^{N}}$, in Appendix 2 we introduce basic definitions of $\mathcal{L}_{\mathcal{M} \mathcal{V}^{N}}$ following $[4,14]$.

For our purpose $\mathcal{L}_{\mathcal{M} \mathcal{V}^{3}}$ is the three-valued logic where $\mathcal{F}$ is defined over the ordered set $\mathcal{N}=\{T, \imath, \perp\}$ and the truth tables given in Table 3. Following [20] 


\begin{tabular}{cc}
$X$ & $\neg$ \\
\hline$T$ & $\perp$ \\
2 & $l$ \\
$\perp$ & $T$ \\
negation
\end{tabular}

\begin{tabular}{llll}
$\wedge$ & $T$ & $l$ & $\perp$ \\
\hline$T$ & $T$ & $l$ & $\perp$ \\
$l$ & $l$ & $l$ & $\perp$ \\
$\perp$ & $\perp$ & $\perp$ & $\perp$ \\
& and & &
\end{tabular}

\begin{tabular}{cccc}
$\rightarrow$ & $T$ & $l$ & $\perp$ \\
\hline$T$ & $T$ & $l$ & $\perp$ \\
$l$ & $T$ & $T$ & $l$ \\
$\perp$ & $T$ & $T$ & $T$ \\
& & implies &
\end{tabular}

\begin{tabular}{cccc}
$\vee$ & $T$ & $l$ & $\perp$ \\
\hline$T$ & $T$ & $T$ & $T$ \\
$l$ & $T$ & $l$ & $l$ \\
$\perp$ & $T$ & $l$ & $\perp$ \\
& or & \\
$\equiv$ & $T$ & $l$ & $\perp$ \\
\hline$T$ & $T$ & $l$ & $\perp$ \\
$l$ & $l$ & $T$ & $l$ \\
$\perp$ & $\perp$ & $l$ & $T$ \\
equivalence &
\end{tabular}

Table 3 The multi-valued logic operators for $\mathcal{L}_{\mathcal{M} \mathcal{V}^{3}}$

we consider $\square$ and $\diamond$ defined in $\mathcal{L}_{\mathcal{M} \mathcal{V}^{3}}$ respectively as:
$\diamond A \equiv \neg A \rightarrow A$
$\square A \equiv \neg(A \rightarrow \neg A)$

Given a marked value $d: C$ with $C \in\{\square \mathbf{G}, \diamond \mathbf{G}, \diamond \mathbf{R}, \square \mathbf{R}\}$, we assume that $d$ belongs to a lattice $\Lambda=(\mathcal{D}, \sqcup . \Pi)$ of values and therefore there exists an order relation $\preceq_{\mathcal{D}}$ on $\mathcal{D}$. An order relation is reflexive, transitive and antisymmetric and is defined as: $\forall a, b \in D, a \preceq_{\mathcal{D}} b \equiv a \sqcap b=a$. We consider the complementary relation $\overline{\preceq_{\mathcal{D}}} \equiv\left\{(a, b) \in \mathcal{D} \times \mathcal{D} \mid \neg\left(a \preceq_{\mathcal{D}} b\right)\right\}$.

Let us consider for each value $d \in \mathcal{D}$ a propositional variable $A \in \mathcal{P}$. Given a valuation $\mathcal{V}$ of $\mathcal{L}_{\mathcal{M} \mathcal{V}^{3}}$ we read: $\mathcal{V}(A)=\mathrm{T}$ as the value $a$ is always reliable; $\mathcal{V}(A)=\{$ as the value $a$ is sometimes reliable; $\mathcal{V}(A)=\perp$ as the value $a$ is never reliable. We define four unary operators $\{\square \mathbf{G}, \diamond \mathbf{G}, \diamond \mathbf{R}, \square \mathbf{R}\}$ to represent in $\mathcal{L}_{\mathcal{M} \mathcal{V}^{3}}$ the characteristic function of sets of values marked homogeneously. Each operator is defined using the Equation 8 and is shown in Table 4. In Figure 2 we show the set diagram for marked values.
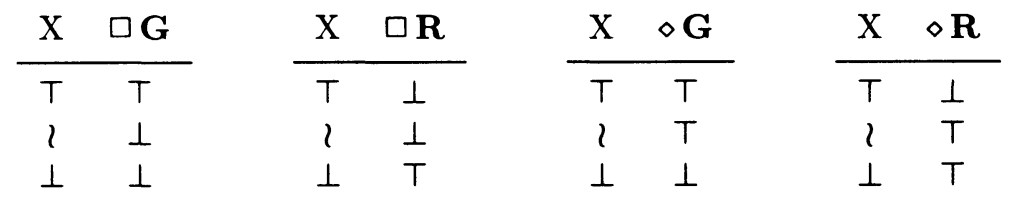

Table 4 The multi-valued interpretation of color operators in $\mathcal{L}_{\mathcal{M} \mathcal{V}^{3}}$

For $o p^{c} \in\{\square \mathbf{G}, \diamond \mathbf{G}, \diamond \mathbf{R}, \square \mathbf{R}\}$ and any propositional variable $t\left[A_{j}\right] \in \mathcal{P}$ it holds in $\mathcal{L}_{\mathcal{M} \mathcal{V}^{3}}: \models_{\mathcal{L}_{\mathcal{M} \mathcal{V}^{3}}}^{\mathcal{V}}\left(o p^{c} A, \top\right)$ if and only if we consider models where $t\left[A_{j}\right]$ belongs to the set of data marked with the marker $C_{j} \equiv o p^{c}$.

While in $\mathcal{L}_{\mathcal{M L}}$ each tuple constraint is a conjunction of quasi-atomic formulas, in $\mathcal{L}_{\mathcal{M} \mathcal{V}^{3}}$ we define tuple constraints as a subset of marked formulas. 


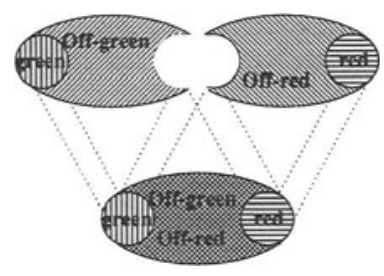

Figure 2 The set diagram for marked value

Definition 6 (Marked formulas) Given a domain $\mathcal{D}$ of values (at least the relations $\{<, \leq,=. \geq,>\}_{\mathcal{D}}$ are defined in $\left.\mathcal{D}\right)$, a set $\mathcal{P}$ of propositional variables (at least one for each value in $\mathcal{D}$ is present in $\mathcal{P}$ ), the set of basic operator $\mathcal{B}=\{\square \mathbf{G}, \diamond \mathbf{G}, \diamond \mathbf{R}, \square \mathbf{R}\} \cup\{\wedge, \vee, \rightarrow, \equiv, \neg\}$ and the set of operators $\mathcal{R}=\{<, \leq,=. \geq,>\}$, marked formulas are:

Marked Atoms: for any propositional variable $A \in \mathcal{P}$ the marked atoms: $\square \mathbf{G} A, \square \mathbf{R} A, \diamond \mathbf{G} A, \square \mathbf{R} A$, are marked formulas;

Relational Atoms: if $A, B$ are marked atoms and $\Theta \in\{<, \leq,=. \geq,>\}$ then $A \Theta B$ is a marked formulas;

Marked Formulas: if $F, G$ are marked formulas then: $\neg F, F \wedge G, F \vee G$, $F \equiv G, F \rightarrow G(F)$ are marked formulas;

nothing else is a marked formula.

Definition 7 (Relational operators) Given two marked atoms $A^{c} \equiv o p_{A}^{c} A$, $B^{c} \equiv o p_{B}^{c} B$, where op c ${ }_{A}^{c}, o p_{B}^{c} \in\{\square \mathbf{G}, \diamond \mathbf{G}, \diamond \mathbf{R}, \square \mathbf{R}\}$ and $A, B \in \mathcal{P}$ correspond to $a, b \in \mathcal{D}$, for any valuation $\mathcal{V}: \mathcal{P} \mapsto\{\top, \imath, \perp\}$ and any $\Theta \in\{<, \leq,=. \geq,>\}$ the valuation $\left(A^{c} \Theta B^{c}\right)^{\mathcal{V}} \equiv\left(A^{c}\right)^{\mathcal{V}} \Theta\left(B^{c}\right)^{\mathcal{V}}$ is:

$$
\begin{array}{ll}
\text { if } \forall_{\mathcal{L}_{\mathcal{M} \mathcal{V}^{3}}}\left(A^{c} \wedge B^{c},\{T\}\right) & \text { then }\left(A^{c} \Theta B^{c}\right)^{\mathcal{V}}=\perp \\
\text { oles if } \forall_{\mathcal{L}_{\mathcal{M} \mathcal{V}^{3}}} A^{c} \wedge B^{c} \equiv A^{c} & \text { then Case of } \models_{\mathcal{L}_{\mathcal{M}} \mathcal{V}^{N}} A^{c} \wedge B^{c} \\
& {[\mathcal{V}(A)=\mathcal{V}(B)=l]:\left(A^{c} \Theta B^{c}\right)^{\mathcal{V}}=1} \\
& {[\mathcal{V}(A)=\mathcal{V}(B) \neq l]:\left(A^{c} \Theta B^{c}\right)^{\mathcal{V}}=\left(a \Theta_{\mathcal{L}_{\mathcal{B}}} b\right)^{\mathcal{D}}} \\
& \text { ondCase } \\
& \left(A^{c} \Theta B^{c}\right)^{\mathcal{V}}=\left(a \Theta_{\mathcal{L}_{\mathcal{B}}} b\right)^{\mathcal{D}}
\end{array}
$$

where $\left(a \Theta_{\mathcal{L}_{\mathcal{B}}} b\right)^{\mathcal{D}}$ is the standard valuation of $\Theta$ over $\mathcal{D}$.

The expression $\left(a \Theta_{\mathcal{L}_{\mathcal{B}}} b\right)^{\mathcal{D}}$ for case $[\mathcal{V}(A)=\mathcal{V}(B) \neq l]$ should be substituted by $\neg\left(a \overline{\Theta_{\mathcal{L}_{\mathcal{B}}}} b\right)^{\mathcal{D}}$ if $\Theta$ is not defined for all $d \in \mathcal{D}$. The symbol $\overline{\Theta_{\mathcal{L}_{\mathcal{B}}}}$ represents the complement of $\Theta_{\mathcal{L}_{\mathcal{B}}}$. We summarize Definition 7 in Table 5. We give notions of satisfiability and validity for marked formulas extending those for $\mathcal{L}_{\mathcal{M} \mathcal{V}^{N}}$

Definition 8 (Color satisfiability) Given a marked formula $F$, a set $U \subset$ $\{T, 2, \perp\}$ and a valuation $\mathcal{V}$ over propositional variables of $F,(F, U)$ is satisfiable (color satisfiable), denoted as $\models_{c}^{\mathcal{V}}(F, U)$, iff $(F)^{\mathcal{V}} \in U$. 


\begin{tabular}{ccccc}
$\Theta_{\mathcal{L}_{\mathcal{M} \mathcal{L}^{3}}}$ & $\square \mathbf{G}$ & $\diamond \mathbf{G}$ & $\diamond \mathbf{R}$ & $\square \mathbf{R}$ \\
\hline$\square \mathbf{G}$ & $\Theta_{\mathcal{L}_{\mathcal{B}}}$ & $\Theta_{\mathcal{L}_{\mathcal{B}}}$ & $\perp$ & $\perp$ \\
$\diamond \mathbf{G}$ & $\neg \Theta_{\mathcal{L}_{\mathcal{B}}}$ & $\Theta_{\mathcal{L}_{\mathcal{B}}}$ & 1 & $\perp$ \\
$\diamond \mathbf{R}$ & $\perp$ & $\mathcal{L}$ & $\Theta_{\mathcal{L}_{\mathcal{B}}}$ & $\neg \bar{\Theta}_{\mathcal{L}_{\mathcal{B}}}$ \\
$\square \mathbf{R}$ & $\perp$ & $\perp$ & $\Theta_{\mathcal{L}_{\mathcal{B}}}$ & $\Theta_{\mathcal{L}_{\mathcal{B}}}$
\end{tabular}

Table 5 The evaluation of $\Theta_{\mathcal{L}_{\mathcal{M} \nu^{3}}} \in\{<, \leq,=. \geq,>\}$ on marked data

Definition 9 (Color validity) Given a marked formula $F$ and a set $U \subset$ $\{\top, \imath, \perp\},(F, U)$ is valid (color valid), denoted as $\models_{c}(F, U)$, iff $\forall \mathcal{V}, \models_{c}^{V} F$.

Note that correspondence between S5 and $\mathcal{L}_{\mathcal{M} \mathcal{V}^{3}}$ holds only considering satisfiability and validity when $U=\{T\}$ (see [20]). Given a marked formula $F$ because the interpretation $(F)^{\mathcal{V}}$ returns a value $v \in\{T, \imath, \perp\}$ we can define at least two different further notions of satisfiability and validity as follows:

Damaged Data Intolerant interpretation $(F)^{\mathcal{V}}=\mathrm{T}$;

Damaged Data Tolerant interpretation $(F)^{\mathcal{V}} \in\{T, l\}$.

We denote with $\models_{\ddagger}^{\mathcal{V}} F, \models_{\ddagger} F$ and $\models_{\dagger}^{\mathcal{V}} F, \models_{\dagger} F$ respectively satisfiability and validity in damaged data intolerant and damaged data tolerant interpretations.

Let us consider a lattice $\Lambda=(\mathcal{D}, \sqcup, \Pi)$, the order relation $\preceq_{\mathcal{D}} \equiv\{(a, b) \in$ $\mathcal{D} \times \mathcal{D} \mid a \sqcap b=a\}$ and the coding of marked values into marked formulas.

Proposition 5 Given $a^{c}, b^{c} \in \mathcal{D}^{c}, a^{c} \equiv a: C_{a}, b^{c} \equiv b: C_{b}$ where $a, b \in \mathcal{D}$ and $C_{a}, C_{b} \in\{\square \mathbf{G}, \diamond \mathbf{G}, \diamond \mathbf{R}, \square \mathbf{R}\}$, the relations $\preceq_{\mathcal{D}^{c}}$ defined as:

$\preceq_{\mathcal{D}^{c}} \equiv\left\{\left(a^{c}, b^{c}\right) \in \mathcal{D}^{c} \times \mathcal{D}^{c} \mid \vDash_{c}\left(\left(A^{c} \leq B^{c}\right),\{T\}\right)\right\}$

is an order relation over the set $\mathcal{D}^{c}$ of marked values.

The Table 6 shows that $\preceq_{D^{c}}$ is an order relation built as the disjunct union of two different order relations.

Definition 10 (Tuple constraint in $\mathcal{L}_{\mathcal{M} \mathcal{V}^{3}}$ ) Given $t^{c} \in \boldsymbol{r}^{c}$ defined on $X\left(A_{1}\right.$, $\left.A_{2}, \ldots, A_{k}\right)$, with $t^{c}=<t^{c}\left[A_{1}\right], t^{c}\left[A_{2}\right], \ldots, t^{c}\left[A_{k}\right]>$, where for each $t^{c}\left[A_{j}\right] \equiv$ $t\left[A_{j}\right]: C_{j}$ it holds $t\left[A_{j}\right] \in \operatorname{dom}\left(A_{j}\right)$ and $C_{j} \in\{\square \mathbf{G}, \diamond \mathbf{G}, \diamond \mathbf{R}, \square \mathbf{R}\}$, the tuple constraint for $t^{c}$ is in $\mathcal{L}_{\mathcal{M} \mathcal{V}^{3}}$ the following conjunction of marked atoms:

$$
\psi^{c}\left(X, t^{c}\right) \equiv \bigwedge_{j=1 \ldots k} \phi^{c}\left(t\left[A_{j}\right]: C_{j}\right)
$$




\begin{tabular}{ccccc}
$\preceq_{\mathcal{D}}$ & $\square \mathbf{G}$ & $\diamond \mathbf{G}$ & $\diamond \mathbf{R}$ & $\square \mathbf{R}$ \\
\hline$\square \mathbf{G}$ & $\preceq_{\mathcal{D}}$ & $\preceq_{\mathcal{D}}$ & $\perp$ & $\perp$ \\
$\circ \mathbf{G}$ & $\neg \preceq_{\mathcal{D}}$ & $\preceq_{\mathcal{D}}$ & $\mathfrak{l}$ & $\stackrel{\perp}{\perp}$ \\
$\diamond \mathbf{R}$ & $\perp$ & $\mathfrak{1}$ & $\preceq_{\mathcal{D}}$ & $\neg \preceq_{\mathcal{D}}$ \\
$\square \mathbf{R}$ & $\perp$ & $\perp$ & $\preceq_{\mathcal{D}}$ & $\preceq_{\mathcal{D}}$
\end{tabular}

Table 6 The partial order relation $\preceq_{\mathcal{D}^{c}}$ on marked data

$$
\begin{array}{llll}
\phi^{c}\left(t\left[A_{j}\right]: \square \mathbf{G}\right) & \equiv \square \mathbf{G} t\left[A_{j}\right] & \phi^{c}\left(t\left[A_{j}\right]: \diamond \mathbf{G}\right) \equiv \diamond \mathbf{G} t\left[A_{j}\right] \\
\phi^{c}\left(t\left[A_{j}\right]: \square \mathbf{R}\right) & \equiv \square \mathbf{R} t\left[A_{j}\right] & \phi^{c}\left(t\left[A_{j}\right]: \diamond \mathbf{R}\right) \equiv \diamond \mathbf{R} t\left[A_{j}\right]
\end{array}
$$

Note that given $t^{c} \in r^{c}$ defined on $X\left(A_{1}, A_{2}, \ldots, A_{k}\right)$ it holds: $\models_{\ddagger}^{\mathcal{V}} \psi^{c}\left(X, t^{c}\right) \equiv \mathcal{M}_{S 5} \vDash_{S 5} \psi^{c}\left(X, t^{c}\right)$ and $\models_{\dagger}^{\mathcal{V}} \psi^{c}\left(X, t^{c}\right) \not \equiv \mathcal{M}_{S 5} \vDash_{S 5} \psi^{c}\left(X, t^{c}\right)$.

\subsection{Evaluating Query Consistency in $\mathcal{L}_{\mathcal{M} \mathcal{V}^{3}}$}

Let us consider $t^{c} \in r^{c}$ defined on $X\left(A_{1}, A_{2}, \ldots, A_{k}\right)$. Suppose that we want know if $t^{c}$ is in the result of a specific $\mathcal{Q}^{c}$ such that $Z=\left(\mathcal{S}\left(\mathcal{Q}^{c}\right) \cap X\right) \neq \emptyset$ where $Z=\left\{A_{i_{1}}, A_{i_{2}}, \ldots, A_{i_{m}}\right\}$ and $Z \subseteq X$. In general for $t^{c}$ it holds: $\psi^{c}\left(X, t^{c}\right) \wedge$ $\sigma_{Q^{c}}\left(t^{c}\right)$ where $\psi^{c}\left(X, t^{c}\right)$ is defined in Equation 5 and $\sigma_{\mathcal{Q}^{c}}\left(t^{c}\right)$ depends from $\mathcal{Q}^{c}$ evaluated on $t^{c} . \psi^{c}\left(X, t^{c}\right) \wedge \sigma_{\mathcal{Q}^{c}}\left(t^{c}\right)$ represents a query consistency constraint, that is the conjunction of two necessary constraints: the former $\psi^{c}\left(X, t^{c}\right) \equiv$ $\left(\bigwedge_{j=1 \ldots k} \phi^{c}\left(t\left[A_{j}\right]: C_{j}\right)\right)$ takes account of color consistency and the latter $\sigma_{Q^{c}}\left(t^{c}\right)$ takes account of the implicitly defined constraint that verifies for each query the existence of values to display.

Definition 11 Given $t^{c} \in r^{c}$ defined on $X\left(A_{1}, A_{2}, \ldots, A_{k}\right)$, with $t^{c}=<$ $t^{c}\left[A_{1}\right], t^{c}\left[A_{2}\right], \ldots, t^{c}\left[A_{k}\right]>$, where for each $t^{c}\left[A_{j}\right] \equiv t\left[A_{j}\right]: C_{j}$ is $t\left[A_{j}\right] \in$ $\operatorname{dom}\left(A_{j}\right)$ and $C_{j} \in\{\square \mathbf{G}, \diamond \mathbf{G}, \diamond \mathbf{R}, \square \mathbf{R}\}$ and $\mathcal{Q}^{c}$ such that $Z=\left(\mathcal{S}\left(\mathcal{Q}^{c}\right) \cap X\right) \neq$ $\emptyset$ where $Z=\left\{A_{i_{1}}, A_{i_{2}}, \ldots, A_{i_{m}}\right\}$, the constraint $\sigma_{\mathcal{Q}^{c}}\left(t^{c}\right)$ is:

$$
\sigma_{\mathcal{Q}^{c}}\left(t^{c}\right) \equiv\left(\bigwedge_{j=1 \ldots m} t\left[A_{i_{j}}\right]\right)
$$

Definition 12 Given $t^{c} \in r^{c}$ defined on $X\left(A_{1}, A_{2}, \ldots, A_{k}\right)$, with $t^{c}=<$ $t^{c}\left[A_{1}\right], t^{c}\left[A_{2}\right], \ldots, t^{c}\left[A_{k}\right]>$, and $\mathcal{Q}^{c}$ such that $Z=\left(\mathcal{S}\left(\mathcal{Q}^{c}\right) \cap X\right) \neq \emptyset, Z=$ $\left\{A_{i_{1}}, A_{i_{2}}, \ldots, A_{i_{m}}\right\}$ and $X \backslash \mathcal{S}\left(\mathcal{Q}^{c}\right)=\left\{A_{l_{1}}, A_{l_{2}}, \ldots, A_{l_{|m-k|}}\right\}$, the query consistency constraint is in $\mathcal{L}_{\mathcal{M V}}$ :

$$
\begin{aligned}
& \Gamma^{c}\left(t^{c}[Z]\right)^{\cap} \wedge \Gamma^{c}\left(t^{c}\left[X \backslash \mathcal{S}\left(\mathcal{Q}^{c}\right)\right]\right)^{\backslash} \\
& \Gamma^{c}\left(t^{c}[Z]\right)^{\cap} \equiv \bigwedge_{j=1 \ldots m} \gamma^{c}\left(t\left[A_{i_{j}}\right]: C_{i_{j}}\right)
\end{aligned}
$$




$$
\begin{aligned}
& \Gamma^{c}\left(t^{c}\left[X \backslash \mathcal{S}\left(\mathcal{Q}^{c}\right)\right]\right)^{\backslash} \equiv \bigwedge_{j=1 \ldots|m-k|} \phi^{c}\left(t\left[A_{l_{j}}\right]: C_{l_{j}}\right)
\end{aligned}
$$

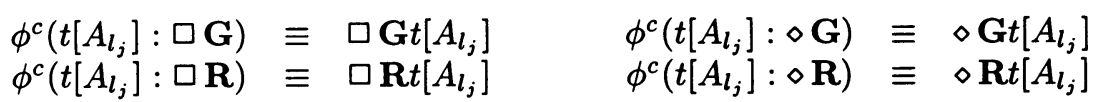

$$
\begin{aligned}
& \gamma^{c}\left(t\left[A_{i_{j}}\right]: \square \mathbf{G}\right) \equiv \square \mathbf{G} t\left[A_{i_{j}}\right] \wedge t\left[A_{i_{j}}\right] \equiv \square \mathbf{G} t\left[A_{i_{j}}\right] \\
& \gamma^{c}\left(t\left[A_{i_{j}}\right]: \square \mathbf{R}\right) \equiv \square \mathbf{R} t\left[A_{i_{j}}\right] \wedge t\left[A_{i_{j}}\right] \equiv \perp \\
& \gamma^{c}\left(t\left[A_{i_{j}}\right]: \diamond \mathbf{G}\right) \equiv \diamond \mathbf{G} t\left[A_{i_{j}}\right] \wedge t\left[A_{i_{j}}\right] \equiv t\left[A_{i_{j}}\right] \\
& \gamma^{c}\left(t\left[A_{i_{j}}\right]: \diamond \mathbf{R}\right) \equiv \diamond \mathbf{R} t\left[A_{i_{j}}\right] \wedge t\left[A_{i_{j}}\right] \equiv \neg t\left[A_{i_{j}}\right] \wedge t\left[A_{i_{j}}\right]
\end{aligned}
$$

Values in a marked tuple can be displayed by a query if at least the subset belonging to the query scheme is not contradictory (see the example below).

Definition 13 (Query consistency) $t^{c}$ defined on $X\left(A_{1}, A_{2}, \ldots, A_{k}\right)$ is query consistent in $\mathcal{L}_{\mathcal{M} \mathcal{V}^{3}}$ with respect to $\mathcal{Q}^{c}$, iff

Damaged Data Intolerant interpretation $\models_{\ddagger}^{\mathcal{V}} \Gamma^{c}\left(t^{c}\left[\mathcal{S}\left(\mathcal{Q}^{c}\right) \cap X\right]\right)^{\cap}$

Damaged Data Tolerant interpretation $\models_{\dagger}^{\mathcal{V}} \Gamma^{c}\left(t^{c}\left[\mathcal{S}\left(\mathcal{Q}^{c}\right) \cap X\right]\right)^{\cap}$

We want manage also situations where user needs $R e d$ values. Therefore, we introduce a constraint $\overline{\Gamma^{c}\left(t^{c}\left[\mathcal{S}\left(\mathcal{Q}^{c}\right) \cap X\right]\right)^{n}}$, dual with respect to Equation 13 and a notion of weak query consistency.

$$
\begin{aligned}
& \overline{\Gamma^{c}\left(t^{c}\left[\mathcal{S}\left(\mathcal{Q}^{c}\right) \cap X\right]\right)^{n}} \equiv \bigwedge_{j=1 \ldots m} \overline{\gamma^{c}\left(t\left[A_{i_{j}}\right]: C_{i_{j}}\right)}
\end{aligned}
$$

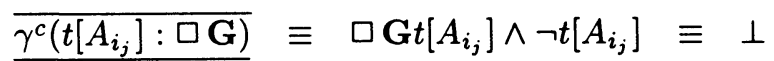

$$
\begin{aligned}
& \overline{\gamma^{c}\left(t\left[A_{i_{j}}\right]: \square \mathbf{R}\right)} \equiv \square \mathbf{R} t\left[A_{i_{j}}\right] \wedge \neg t\left[A_{i_{j}}\right] \equiv \square \mathbf{R} t\left[A_{i_{j}}\right]
\end{aligned}
$$

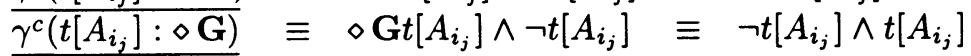

$$
\begin{aligned}
& \overline{\gamma^{c}\left(t\left[A_{i_{j}}\right]: \diamond \mathbf{R}\right)} \equiv \diamond \mathbf{R} t\left[A_{i_{j}}\right] \wedge \neg t\left[A_{i_{j}}\right] \equiv \neg t\left[A_{i_{j}}\right]
\end{aligned}
$$

Definition 14 (Weak query consistency) $t^{c}$ defined on $X\left(A_{1}, A_{2}, \ldots\right.$, $A_{k}$ ) is weakly query consistent in $\mathcal{L}_{\mathcal{M} \mathcal{V}^{3}}$ with respect to $\mathcal{Q}^{c}$, iff

Damaged Data Intolerant interpretation $\models_{\ddagger}^{\mathcal{V}} \overline{\Gamma^{c}\left(t^{c}\left[\mathcal{S}\left(\mathcal{Q}^{c}\right) \cap X\right]\right)^{n}}$
Damaged Data Tolerant interpretation $\models_{\dagger}^{\mathcal{V}} \overline{\Gamma^{c}\left(t^{c}\left[\mathcal{S}\left(\mathcal{Q}^{c}\right) \cap X\right]\right)^{n}}$

\section{RELATIONAL ALGEBRA FOR MARKED DATA}

The semantics given so far state, for a marked tuple, how each of its marked values individually constraints the behavior of a marked database. From the 
given semantics of color markers follow sufficient conditions to test when a marked tuple belongs to a marked query. This test allows us to build practical marked database systems on top of traditional systems.

\subsection{Conditional Expressions}

Definition 15 (Propositional formulas over marked databases) Let $\boldsymbol{r}^{\boldsymbol{c}}$ defined over $X\left(A_{1}, A_{2}, \ldots, A_{k}\right)$, a propositional formula $\mathcal{F}$ over $X$ is:

Atoms: $A_{i} \Theta A_{j}$ or $A_{i} \Theta c$ are propositional formulas over $X$; where $A_{i}, A_{j} \in$ $X, c \in \operatorname{dom}(X)$ (then $c$ is not a marked datum) and $\Theta \in\{<, \leq,=. \geq,>\}$; Formulas: if $\mathcal{F}, \mathcal{E}$ are formulas on $X$ then: $\neg(\mathcal{F}), \mathcal{F} \wedge \mathcal{E}, \mathcal{F} \vee \mathcal{E},(\mathcal{F})$ are formulas on $X$;

nothing else is a formula on $X$.

A propositional formula $\mathcal{F}$ over $X$ associates a value in $\{T, l, \perp\}$ with each marked tuple. The effective evaluation is obtained for each tuple $t^{c} \in r^{c}$ coding $\mathcal{F}$ as a marked formula.

Definition 16 (Propositional formulas coding) Given $t^{c} \in \boldsymbol{r}^{c}$ defined on $X\left(A_{1}, A_{2}, \ldots, A_{k}\right)$, with $t^{c}=<t^{c}\left[A_{1}\right], t^{c}\left[A_{2}\right], \ldots, t^{c}\left[A_{k}\right]>$, where for each $t^{c}\left[A_{j}\right] \equiv t\left[A_{j}\right]: C_{j}$ is $t\left[A_{j}\right] \in \operatorname{dom}\left(A_{j}\right)$ and $C_{j} \in\{\square \mathbf{G}, \diamond \mathbf{G}, \diamond \mathbf{R}, \square \mathbf{R}\}$; given a propositional formula $\mathcal{F}$ over $X$, the coding function ()$^{\left(t^{c}, X\right)}$ that maps $\mathcal{F}$ into a marked formula is:

$\begin{array}{lllll}\mathcal{F} & \\ (c)^{\left(t^{c}, X\right)} & \equiv \square \text { marked formula is: } & \left(A_{i} \Theta A_{j}\right)^{\left(t^{c}, X\right)} & \equiv\left(A_{i}\right)^{\left(t^{c}, X\right)} \Theta\left(A_{j}\right)^{\left(t^{c}, X\right)} \\ \left(A_{k}\right)^{\left(t^{c}, X\right)} & \equiv \phi^{c}\left(t\left[A_{k}\right]: C_{k}\right) & \left(A_{i} \Theta c\right)^{\left(t^{c}, X\right)} & \equiv\left(A_{i}\right)^{\left(t^{c}, X\right)} \Theta(c)^{\left(t^{c}, X\right)} \\ (\neg(\mathcal{F}))^{\left(t^{c}, X\right)} & \equiv \neg(\mathcal{F})^{\left(t^{c}, X\right)} & (\mathcal{F} \wedge \mathcal{E})^{\left(t^{c}, X\right)} & \equiv(\mathcal{F})^{\left(t^{c}, X\right)} \wedge(\mathcal{E})^{\left(t^{c}, X\right)} \\ ((\mathcal{F}))^{\left(t^{c}, X\right)} & \equiv(\mathcal{F})^{\left(t^{c}, X\right)} & (\mathcal{F} \vee \mathcal{E})^{\left(t^{c}, X\right)} & \equiv(\mathcal{F})^{\left(t^{c}, X\right)} \vee(\mathcal{E})^{\left(t^{c}, X\right)}\end{array}$

where $A_{i}, A_{j} \in X, c \in \operatorname{dom}(X), \phi^{c}\left(t\left[A_{k}\right]: C_{k}\right)$ is in Equation $11, \Theta \in\{<, \leq$ $,=. \geq,>\}$ is in Definition 7 and $\{\wedge, \vee, \rightarrow, \equiv, \neg\}$ are in Table 3 .

We consider in $\mathcal{L}_{\mathcal{M} \mathcal{V}^{3}}$ those valuations $\mathcal{V}$ such that: $\forall_{j=1 \ldots k} \models_{c}^{\mathcal{V}}\left(\phi^{c}\left(t\left[A_{k}\right]\right.\right.$ : $\left.\left.C_{k}\right),\{T\}\right)$ and given $U \subset\{T, l, \perp\}$, we denote satisfiability of $F$ as: $t^{c} \models_{c}$ $(F, U) \equiv \models_{c}^{\mathcal{V}\left(t^{c}\right)}(F, U)$. Given $t^{c} \in \boldsymbol{r}^{c}$ defined on $X$ and given $F=(\mathcal{F})^{\left(t^{c}, X\right)}$ (more in general, any $F \in \mathcal{L}_{\mathcal{M} \mathcal{V}^{3}}$ ), we have:

\section{Damaged Data Intolerant interpretation $t^{c} \models_{\ddagger} F$ iff $\models_{\ddagger}^{\mathcal{V}\left(t^{c}\right)} F$ Damaged Data Tolerant interpretation $t^{c} \models_{\dagger} F$ iff $\models_{\dagger}^{\mathcal{V}\left(t^{c}\right)} F$}

Each of them represents a different user interpretation of the reliability of marked data. Moreover, for each query the user can choose between different notions of query consistency. 


\subsection{Algebraic Operators}

Because a single tuple $t$ defined on $R\left(B_{1}, B_{2}, \ldots, B_{m}\right)$ in a standard database can be obtained from $4^{m}$ different marked tuples, a grouping operator $\mathcal{G}^{c}$ for marked tuples that differ only for color markers is necessary. Moreover, each marked tuple must satisfy a query consistency constraint to belong to the result of a query and this gives rise to different query tests $\mathcal{T}_{(a, I)}^{c}$.

The pre-result is a wide set of marked tuples from which through grouping operation and query test all unnecessary and contradictory tuples are removed. The high-quality technology used to optimize standard queries suggests us to calculate the per-result, as much as possible, using standard systems. We consider for each algebraic operator $\left\{\cap, \cup, \backslash, \times, \bowtie, \pi_{X}, \sigma_{\mathcal{F}}\right\}$ a different pre-result operator $\left\{\cup, \cap, \backslash, \times\right.$, pre- $\bowtie_{=}^{c}$, pre- $\bowtie_{\Theta}^{c} \pi_{X}$, pre- $\left.\sigma_{\mathcal{F}}^{c}\right\}$ and different query tests $\mathcal{T}_{(a, I)}^{c}$ where $I \in\{\ddagger, \dagger\}$ and $a \in\{\star, \sim\}((\star)$ normal, $(\sim)$ weak query consistency). The grouping operator $\mathcal{G}^{c}$ is unique, even if it depends on the chosen interpretation through $\mathcal{T}_{(a, I)}^{c}$.

Definition 17 (The query test $\left.\mathcal{T}_{(a, I)}^{c}\right)$ Given $t^{c} \in r^{c}$ defined on $X\left(A_{1}\right.$, $\left.A_{2}, \ldots, A_{k}\right)$ and a set of attributes $S$ such that $Z=(S \cap X) \neq \emptyset, \mathcal{T}_{(a, I)}^{c}\left(t^{c}, X, S\right)$ is defined as shown in Table 7.

\begin{tabular}{cllll}
\hline $\mathcal{T}_{(a, I)}^{c}$ & & $\ddagger$ & & $\dagger$ \\
\hline weak: & $t^{c} \models_{\ddagger}$ & $\overline{\Gamma^{c}\left(t^{c}[Z]\right)^{n}}$ & $t^{c} \models_{\dagger}$ & $\overline{\Gamma^{c}\left(t^{c}[Z]\right)^{n}}$ \\
normal: & $t^{c} \models_{\ddagger}$ & $\Gamma^{c}\left(t^{c}[Z]\right)^{n}$ & $t^{c} \models_{\dagger}$ & $\Gamma^{c}\left(t^{c}[Z]\right)^{n}$ \\
\hline
\end{tabular}

Table 7 The Table of $\mathcal{T}_{(a, I)}^{c}$

Note that $\mathcal{T}_{(a, I)}^{c}$ is a meta logic operator that returns values in $\{\top, \perp\}$.

Proposition 6 Given $t^{c} \in r^{c}$ defined on $X\left(A_{1}, A_{2}, \ldots, A_{k}\right)$ and given a specific $\mathcal{Q}^{c}$ such that $Z=\left(\mathcal{S}\left(\mathcal{Q}^{c}\right) \cap X\right) \neq \emptyset$, $t^{c}$ is valid with respect to $\mathcal{T}_{(a, I)}^{c}$ iff $t^{c}$ has over attributes in $Z$ no conflicting forms (see Equation 7) and case of:

$a=\star, I=\ddagger, t^{c}$ has all green and/or Off-green data (3 cases);

$a=\star, I=\dagger, t^{c}$ has all green and/or Off-green and/or Off-red data (7 cases);

$a=\sim, I=\ddagger, t^{c}$ has all red and/or Off-red data (3 cases);

$a=\sim, I=\dagger, t^{c}$ has all red and/or Off-red and/or Off-green data (7 cases);

Example 1 Let us consider relations in Table 2 and a query that projects the 
first and the last column of person-room-telephone. In Table 11 (appearing in Appendix 3) we show the query result with respect to weak and normal query consistency.

Definition 18 (The grouping operator $\left.\mathcal{G}^{c}\right)$ Given $t^{c}$ defined over $X\left(A_{1}\right.$, $A_{2}, \ldots, A_{k}$ ) such that the corresponding relation $t$ is a single tuple $t$, the grouping operator $\mathcal{G}^{c}\left(\boldsymbol{t}^{c}\right)$ is:

$$
\begin{aligned}
& \text { if } \quad \exists t^{c} \in t^{c} \text { s.t. } \forall t^{\prime c} \in t^{c}\left(t^{\prime c} \neq t^{c} \rightarrow t^{\prime c} \ll X t^{c}\right) \\
& \mathcal{G}^{c}\left(t^{c}\right) \equiv \text { then } \mathcal{G}^{c}\left(t^{c}\right)=t^{c} \\
& \text { else } \mathcal{G}^{c}\left(t^{c}\right)=t \text { with all markers } C_{j} \equiv \diamond \mathbf{G}(j=1 \ldots k)
\end{aligned}
$$

where $t^{\prime c} \neq t^{c}$ means that $t^{\prime c}, t^{c}$ differ at least for a marker and $t^{\prime c} \ll_{X} t^{c}$ is: Caseof $\left\langle\mathcal{T}_{(a, I)}^{c}\left(t^{\prime c}, X, X\right), \mathcal{T}_{(a, I)}^{c}\left(t^{c}, X, X\right)>\right.$

$$
\begin{array}{ll}
<\top, T>: & \left(\left(\operatorname{num}\left(\square \mathbf{G}, t^{\prime c}\right)=\operatorname{(num}\left(\square \mathbf{G}, t^{\prime c}\right)<\operatorname{num}\left(\square \mathbf{G}, t^{c}\right)\right) \vee\right. \\
\left.\left.<\mathrm{G}, t^{c}\right)\right) \wedge\left(\operatorname{num}\left(\diamond \mathbf{G}, t^{\prime c}\right)<\right. \\
<\top, \perp>: & \perp \\
<\perp, T>: & \mathrm{T} \\
<\perp, \perp>: & \perp
\end{array}
$$

\section{EndCase}

where num $\left(C, t^{c}\right)$ counts the number of markers $C$ in $t^{c}$.

Note that, because of $\mathcal{T}_{(a, I)}^{c}, \mathcal{G}^{c}$ and $\ll_{X}$ depend on which interpretation the user adopts. The grouping of a marked relation is obtained by applying $\mathcal{G}^{c}$ to each group of tuples identical in value but different in markers. Note that for a group formed by only one tuple $\mathcal{G}^{c}$ returns the tuple itself.

The set of basic pre-operators for marked relations, $\times, \cup, \cap, \backslash$ and $\pi_{X}$ coincides with standard operators of relational algebra, considering marked values as atomic values. Let us introduce a standard renaming operator $\mu_{X \mapsto Y}\left(r^{\boldsymbol{c}}\right)$ that renames attributes $X$ of $r^{c}$ in attributes $Y$ and the operator $\Delta_{F}\left(r^{c}\right)$.

Definition 19 (Three-valued satisfiable set) Given $\boldsymbol{r}^{c}$ defined over $X\left(A_{1}\right.$, $\left.A_{2}, \ldots, A_{k}\right)$ and $F \in \mathcal{L}_{\mathcal{M} \mathcal{V}^{3}}$, the three valued satisfiable set $\Delta_{F}\left(r^{c}\right)$ is: $\Delta_{F}\left(\boldsymbol{r}^{c}\right) \equiv\left\{t^{c} \in \boldsymbol{r}^{c} \mid t^{c} \vDash_{I} F\right\}$

$\Delta_{F}$ is useful to evaluate $\mathcal{T}_{(a, I)}^{c}$. In fact, each expression showed in Table 7 is calculated as $\Delta_{\mathcal{T}_{(a, I)}^{c}(X, Y)}$, where $X, Y$ are sets of attributes.

Definition 20 (Marked selection $\sigma_{\mathcal{F}}^{c}$ ) Given $r^{c}$ defined over $X\left(A_{1}, A_{2}\right.$, $\left.\ldots, A_{k}\right)$ and $\mathcal{F}$ defined over $C \subseteq X$, the pre-selection pre- $\sigma_{\mathcal{F}}^{c}$ is: pre- $\sigma_{\mathcal{F}}^{c}\left(r^{c}\right) \equiv\left\{t^{c} \in r^{c} \mid \mathcal{T}_{(a, I)}^{c}\left(t^{c}, X, C\right)\right.$ and $\left.\left(t^{c} \vDash_{I}(\mathcal{F})^{\left(t^{c}, X\right)}\right)\right\}$ pre- $\sigma_{\mathcal{F}}^{c}\left(r^{c}\right) \equiv \Delta_{\mathcal{T}_{(a, I)}^{c}(X, C)}\left(r^{c}\right) \cap \Delta_{(\mathcal{F})^{\left(t^{c}, X\right)}}\left(r^{c}\right)$ and the marked selection $\sigma_{\mathcal{F}}^{c}$ is: $\sigma_{\mathcal{F}}^{c}\left(\boldsymbol{r}^{c}\right) \equiv \mathcal{G}^{c}\left(\right.$ pre- $\left.\sigma_{\mathcal{F}}^{c}\left(\boldsymbol{r}^{c}\right)\right) \equiv \mathcal{G}^{c}\left(\Delta_{\mathcal{T}_{(a, I)}^{c}(X, C)}\left(\boldsymbol{r}^{c}\right) \cap \Delta_{\left.(\mathcal{F})^{(t}, X\right)}\left(\boldsymbol{r}^{c}\right)\right)$

Example 2 (Marked selection $\sigma_{\mathcal{F}}^{c}$ ) Let us consider relation view defined 
on $X=\{$ ssN, BILL, LAST-BILL $\}$ and showed in Table 13. $\sigma_{\mathcal{F}}^{c}$ where $\mathcal{F}=$ BILL $>$ LAST-BILL under different interpretations is shown in Table 12 and the corresponding query constraints are shown in Table 14.

Definition 21 (Marked projection $\pi_{Y}^{c}$ ) Given $r^{c}$ defined over $X\left(A_{1}, A_{2}\right.$, $\left.\ldots, A_{k}\right)$ and $Y \subseteq X$, the marked projection $\pi_{Y}^{c}$ is:

$\pi_{Y}^{c}\left(\boldsymbol{r}^{c}\right) \equiv \mathcal{G}^{c}\left(\Delta_{\mathcal{T}_{(a, I)}^{c}(Y, Y)}\left(\pi_{Y}\left(\boldsymbol{r}^{c}\right)\right)\right) \equiv\left\{t^{c}[Y] \mid t^{c} \in \boldsymbol{r}^{c}\right.$ and $\left.\mathcal{T}_{(a, I)}^{c}\left(t^{c}, Y, Y\right)\right\}$

Definition 22 (Marked natural Join $\bowtie_{=}^{c}$ ) Given $p^{c}$, $\boldsymbol{q}^{\boldsymbol{c}}$ defined respectively on $P\left(A_{1}, A_{2}, \ldots, A_{m}\right)$ and $Q\left(B_{1}, B_{2}, \ldots, B_{n}\right)$, such that $P \cap Q=X$, the natural pre-Join pre- $\bowtie_{=}^{c}$ is:

$\boldsymbol{p}^{\boldsymbol{c}}$ pre- $\bowtie_{=}^{c} \boldsymbol{q}^{\boldsymbol{c}} \equiv \pi_{P \cup Q}\left(\right.$ pre- $\left.\sigma_{\mathcal{X}=\mathcal{Y}}^{c}\left(\left(\boldsymbol{p}^{\boldsymbol{c}} \times \mu_{X \mapsto Y}\left(\boldsymbol{q}^{\boldsymbol{c}}\right)\right) \cup\left(\mu_{X \mapsto Y}\left(\boldsymbol{p}^{\boldsymbol{c}}\right) \times \boldsymbol{q}^{\boldsymbol{c}}\right)\right)\right)$ where $P \cup Q$ is the scheme for pre- $\bowtie \stackrel{c}{=}, Y \nsubseteq(P \cup Q), X=Y$ stands for the expression that equates attributes $X$ and $Y$. The marked natural Join is:

$\boldsymbol{p}^{c} \bowtie_{=}^{c} \boldsymbol{q}^{c} \equiv \mathcal{G}^{c}\left(\Delta_{\mathcal{T}_{(a, I)}^{c}(P \cup Q, P \cup Q)}\left(\boldsymbol{p}^{c}\right.\right.$ pre- $\left.\left.\bowtie_{=}^{c} \boldsymbol{q}^{c}\right)\right) \equiv \pi_{P \cup Q}^{c}\left(\boldsymbol{p}^{c}\right.$ pre- $\left.\bowtie_{=}^{c} \boldsymbol{q}^{c}\right)$

Note that, following Table 5, in case of $\Theta \equiv "{ }^{\prime \prime}$ and damaged data tolerant interpretation the expression $X=Y$ equates attributes that are different in value. Therefore, the projection $\pi_{P \cup Q}$ can return $2^{|X|}$ different tuples for each $t^{c} \in$ pre- $\sigma_{\mathcal{X}=\mathcal{Y}}^{c}\left(\left(\boldsymbol{p}^{c} \times \mu_{X \mapsto Y}\left(\boldsymbol{q}^{c}\right)\right) \cup\left(\mu_{X \mapsto Y}\left(\boldsymbol{p}^{c}\right) \times \boldsymbol{q}^{c}\right)\right)$ that has different values between the equate attributes. The union of two products is necessary to manage this case (see the following example).

Example 3 (Marked natural Join $\bowtie_{=}^{c}$ ) Let us consider relations PAYMENT and TELEPHONE of Tables 1 and 2. pre- $\bowtie_{=}^{c}$ with respect to normal query consistency and different interpretations is shown in Tables 15 and $16 . \bowtie_{=}^{c}$ is shown in Tables 17 and 18.

Definition 23 (Marked $\Theta$-Join $\bowtie_{\Theta}^{c}$ ) Given $\boldsymbol{p}^{\boldsymbol{c}}, \boldsymbol{q}^{\mathbf{c}}$ defined respectively on $P\left(A_{1}, A_{2}, \ldots, A_{m}\right)$ and $Q\left(B_{1}, B_{2}, \ldots, B_{n}\right)$, such that $P \cap Q=\emptyset$ and given $\Theta$ defined over $C \subseteq(P \cup Q)$, the $\Theta$-pre-Join pre- $\bowtie_{\Theta}^{c}$ is:

$p^{c}$ pre- $\bowtie_{\Theta}^{c} q^{c} \equiv p r e-\sigma_{\Theta}^{c}\left(p^{c} \times q^{c}\right)$

where $P \cup Q$ is the scheme for pre- $\bowtie_{\Theta}^{c}$ and the marked $\Theta$-Join is:

$\boldsymbol{p}^{c} \bowtie_{\Theta}^{c} \boldsymbol{q}^{c} \equiv \mathcal{G}^{c}\left(\Delta_{\mathcal{T}_{(a, I)}^{c}(P \cup Q, P \cup Q)}\left(\boldsymbol{p}^{c}\right.\right.$ pre- $\left.\left.\bowtie_{\Theta}^{c} \boldsymbol{q}^{c}\right)\right) \equiv \pi_{P \cup Q}^{c}\left(\boldsymbol{p}^{c}\right.$ pre- $\left.\bowtie_{\Theta}^{c} \boldsymbol{q}^{c}\right)$

Note that following Table 5 and damaged data tolerant interpretation the expression $\Theta$ holds between not correctly related values. Therefore, the projection $\pi_{P \cup Q}$ returns tuples that never should be considered in a standard query (see the following example).

Example 4 (Marked $\Theta-J o i n ~ \bowtie_{\Theta}^{c}$ ) Let us consider relations TELEPHone and PAYMENT of Tables 1 and 2 and dot notation as a renaming for attribute TELEPHONE of both tables. Consider $\Theta=$ TELEPHONE. TELEPHONE $\leq$ PAYMENT. TELEPHONE. 
pre- $\aleph_{\Theta}^{c}$ with respect to normal query consistency and different interpretations is shown in Tables 19 and 20. $\aleph_{\Theta}^{c}$ is shown in Tables 21 and 22.

For all other algebra operators $\left\{x^{c}, \cup^{c}, \cap^{c}, \backslash^{c}\right\}$ their definition is:

Definition 24 Given $\boldsymbol{p}^{c}, \boldsymbol{q}^{c}$ defined over suitable schemes $P\left(A_{1}, A_{2}, \ldots\right.$, $\left.A_{m}\right)$ and $Q\left(B_{1}, B_{2}, \ldots, B_{n}\right)$ each op $p^{c} \in\left\{x^{c}, \cup^{c}, \cap^{c}, \backslash^{c}\right\}$ is:

$\boldsymbol{p}^{c} o p^{c} \boldsymbol{q}^{c} \equiv \mathcal{G}^{c}\left(\pi_{P \cup Q}\left(\boldsymbol{p}^{c} p r e-o p^{c} \boldsymbol{q}^{c}\right)\right) \equiv \pi_{P \cup Q}^{c}\left(\boldsymbol{p}^{c} p r e-o p^{c} \boldsymbol{q}^{c}\right)$

All operators of the algebra for marked relations are defined using standard $\cup, \cap, \times, \backslash, \pi_{X}$ and two extra operators: $\Delta_{F}$ and $\mathcal{G}^{c}$. That is: a management system for marked relations can be built on top of a standard database management system augmented with three-valued propositional formulas.

In [4] it is shown that satisfiability of formulas in conjunctive normal form for three-valued propositional logics is decidable in polynomial time, under the assumption of a fixed theory and considering as input the formula.

Proposition 7 The evaluation problem for a query defined using operators provided for the algebra of marked relations belongs to the same computational complexity class of the evaluation problem for standard relational algebra.

\section{RELATED WORK}

Two main research areas are related to the problem addressed in this work, namely Generalized Annotated Logic Programming and Null Values Theory.

The theory of Generalized Annotated Logic Programming (GAP) by Kifer and Subrhaniam [16] has been proposed to deal with inconsistencies in knowledge bases. The utility of annoted logics for reasoning with inconsistency and for programming expert systems has been also argued [16]. The expressive power of GAP is very rich and it subsumes also some temporal logic programming, but GAP cannot be adequately implemented [16]. As remarked by $\mathrm{Lu}$ [19] GAP theory is strongly related with a first-order logic of signed formulas and with the labeled deductive system proposed by Gabbay [9]. All of these researches involve studies of multi-valued, standard and modal logics [19]. Our solution aims to use only formulas expressed by suitable propositional logics instead of more expressive predicate logics. We lack in expressive power but we gain in tractability, as is shown in Section 5. In any case we do not lack in expressive power about user expectations. The main difference between our proposal and one provided by a logic programming with signs and annotations is that we consider signed constants instead of signed formulas. A mapping of our proposal in GAP is possible but it does not provide either new insights in the problem or better implementation of our solution.

The null values theory (see $[3,5]$ ) introduces a set of explicit markers to 
manage unknown values. The meaning of markers for null values is shown in Table 8 where: $\perp$ denotes a non-existent value; $D$ denotes an attribute domain and it is used to say that a value exists but it is unknown; $D \cup \perp$ denotes an unknown value that lies either on the domain $D$ or $\perp ; V_{x} \cup \perp$ says that the actual value can lie either in the set $V_{x} \subseteq D$ or $\perp$; and $V_{x}$ says that the value is in the set $V_{x} \subseteq D$ and $\{v\}$ denotes the specified value. The null values theory lacks one of the basic requirements for databases with explicit markings of damaged data, that is: in any case a single value is provided and stored in the database. We have considered two possible mappings of color markers into null values markers, as shown in Table 9. Nevertheless, these mappings cannot represent that a value is always provided by the defender or by the offender. Moreover, standard rules for the management of null values are too restrictive (see [5]) and therefore the null values theory cannot give a satisfactory solution for the management of databases with explicit markings of damaged data.

\begin{tabular}{lclc}
\hline Null Marker & Semantics & Null Marker & Semantics \\
\hline pl_mark & $\perp$ & ex_mark & $D$ \\
\hline ma_mark & $D \cup \perp$ & pm_mark $\left(V_{x}\right)$ & $V_{x} \cup \perp$ \\
\hline pa_mark $\left(V_{x}\right)$ & $V_{x}$ & va_mark $(v)$ & $\{v\}$ \\
\hline
\end{tabular}

Table 8 Null Values Markers

\begin{tabular}{|c|c|c|c|c|c|}
\hline \multicolumn{3}{|c|}{ I mapping } & \multicolumn{3}{|c|}{ II mapping } \\
\hline$\square \mathbf{R}$ & $\mapsto$ & pl_mark & $\square \mathbf{R}$ & $\mapsto$ & pl_mark \\
\hline$\diamond \mathbf{R}$ & $\mapsto$ & ex_mark & $\diamond \mathbf{R}$ & $\mapsto$ & ma_mark \\
\hline$\diamond \mathbf{G}$ & $\mapsto$ & $p a_{-} \operatorname{mark}\left(V_{x}\right)$ & $\diamond \mathbf{G}$ & $\mapsto$ & $p m_{-} \operatorname{mark}\left(V_{x}\right)$ \\
\hline$\square \mathbf{G}$ & $\mapsto$ & $v a \_m a r k(v)$ & $\square \mathbf{G}$ & $\mapsto$ & $v a_{-} \operatorname{mark}(v)$ \\
\hline
\end{tabular}

Table 9 Mappings of marked data using null values

\section{CONCLUSIONS}

Maintaining absolute integrity defies the best efforts of system designers, and so approaches based on a black-and-white view of integrity necessarily invite problems. Fortunately, researchers are developing techniques to recognize and even exploit deviations in integrity. 
Our proposal aims to deal with the general problem of relaxing integrity constraints in databases following a tractable approach. We use the large set of developed theory for nonstandard logics to cut out a small, reasonable framework for solving our problem in an effective way. Nevertheless, our proposal is not the final solution to the problem; it lacks the complete automation of damaged data management. Moreover, our framework is rigidly defined for a markers scheme of four colors and cannot be extended to arbitrary sets of markers. To obtain a general solution, further investigations in the addressed areas of multivalued and signed logics should be done.

Results provided in this paper can be considered as preliminary for a complete foundation of marked databases. With our proposal we aim to have shown a pragmatic and reasonable approach for dealing with, in general, an intractable problem, without lacking in theoretical foundation.

In future works we aim to study other relevant problems for database with explicit markings of damaged data, as the management of functional dependencies, integrity constraints, and query optimization.

\section{Acknowledgement}

This work was performed while Giovanni Rumolo was visiting the Center for Secure Information Systems at George Mason University. The work of Sushil Jajodia was supported by Joe Giordano of Air Force Research Laboratory/Rome. Jajodia is also with the Center for Secure Information Systems, George Mason University, Fairfax, VA 22030-4444, USA.

\section{REFERENCES}

[1] P. Ammann, S. Jajodia, C. D. McCollum, and B. T. Blaustein. Surviving information warfare attacks on databases. In Proceedings of the 1997 IEEE Symposium on Security and Privacy, 1997.

[2] P. Ammann, S. Jajodia, and I. Ray. Applying formal methods to semantic-based decomposition of transactions. ACM Transactions on Database Systems, 22(2):215-254, June 1997.

[3] P. Atzeni and V. D. Antonellis. Relational Database Theory. The Benjamin/Cummings Publishing Company, Inc., Redwood City, California, 1993.

[4] M. Cadoli and M. Schaerf. On the complexity of entailment in propositional multivalued logics. Annals of Mathematics and Artificial Intelligence, 18(1):29-50, 1996.

[5] K. S. Candan, J. Grant, and V. S. Subrahmanian. A unified treatment of null values using constraints. Technical Report CS-TR-3456, University of Maryland, College Park, Apr. 1995.

[6] A. K. Chandra. Theory of Database Queries. In Proceedings of the 
Seventh ACM Symposium on Principles of Database Systems, pages 1-9, Austin, Texas, Mar. 1988.

[7] P. Chisholm, G. Chen, D. Ferbrache, P. Thanisch, and M. H. Williams. Coping with indefinite and negative data in deductive databases: A survey. Data \& Knowledge Engineering, 2:259-284, 1987.

[8] C. J. Date. Null Values in Database Management, chapter 15, pages 313-334. Addison-Wesley, Reading, MA, 1986.

[9] D. M. Gabbay. Labelled Deductive Systems, volume 1. Oxford University Press, 1996.

[10] H. García-Molina. Using semantic knowledge for transaction processing in a distributed database. ACM Transactions on Database Systems, 8(2):186-213, June 1983.

[11] R. Goldblatt. Logics of Time and Computation, Second Edition, Revised and Expanded, volume 7 of CSLI Lecture Notes. CSLI, Stanford, 1992 (first edition 1987). Distributed by University of Chicago Press.

[12] J. Grant. Null values in a relational database. Information Processing Letters, 6(5):156-157, Oct. 1977.

[13] R. Hähnle. Automated Deduction in Multiple-Valued Logics, volume 10 of International Series of Monographs on Computer Science. Oxford University Press, 1994. Reviewed in Computing Reviews, August 1995, \#9508-0571 and Notre Dame Journal of Formal Logic, 37(4), Fall 1996.

[14] R. Hähnle and G. Escalada-Imaz. Deduction in many-valued logics: a survey. Mathware \& Soft Computing, IV(2):69-97, 1997.

[15] G. E. Hughes and M. J. Cresswell. An Introduction to Modal Logic. Methuen \& Co. Ltd, London, 2 edition, 1974.

[16] M. Kifer and V. S. Subrahmanian. Theory of generalized annotated logic programming and its applications. The Journal of Logic Programming, 12(1 and 2):335-367, Jan. 1992.

[17] N. Krishnakumar and A. J. Bernstein. Bounded ignorance: A technique for increasing concurrency in a replicated system. ACM Transactions on Database Systems, 19(4):586-625, Dec. 1994.

[18] K. C. Liu and R. Sunderraman. Indefinite and maybe information in relational databases. ACM Transactions on Database Systems, 15(1):1-39, Mar. 1990.

[19] J. J. Lu. Logic programming with signs and annotations. Journal of Logic and Computation, 6(6):755-778, Dec. 1996.

[20] G. Malinowski. Many-Valued Logics, volume 25 of Oxford Logic Guides. Oxford University Press, 1993.

[21] V. S. Subrahmanian. Paraconsistent disjunctive deductive databases. In Proceedings of the 20th International Symposium on Multiple-Valued Logic, pages 339-346, Charlotte, NC, May 1990.

[22] M. Y. Vardi. Why is modal logic so robustly decidable? Technical Report TR97-274, Rice University, Apr. 12, 1997. 


\section{APPENDIX 1 MODAL LOGIC}

The language $\mathcal{L}_{\mathcal{M} \mathcal{C}}$ is defined extending propositional logic with two additional symbols: $\square, \diamond$. Given a set $\mathcal{P}$ of propositional variables, $\mathcal{L}_{\mathcal{M} \mathcal{L}}$ formulas are defined as:

Atoms: every $A \in \mathcal{P}$ and the two constants $\mathrm{T}, \perp$ are atomic propositional formulas of $\mathcal{L}_{\mathcal{M L}}$

Formulas: if $A, B$ are formulas then: $\neg A, A \wedge B, A \vee B, A \rightarrow B,(A), \square A, \diamond A$ are formulas of $\mathcal{L}_{\mathcal{M L}}$;

nothing else is a formula of $\mathcal{L}_{\mathcal{M} \mathcal{L}}$.

Definition 25 (Quasi-atomic formulas) If $A \in \mathcal{P} \cup\{\top, \perp\}$ is an atomic formula then $\square A, \diamond A, \square \neg A, \diamond \neg A$ are quasi atomic formulas of $\mathcal{L}_{\mathcal{M} \mathcal{C}}$.

The two symbols $\square, \diamond$ can be read in different ways, common readings of them are: necessity and possibility: $\square A \equiv$ "it is necessary that $A$ is true", $\diamond A \equiv$ "it is possible that $A$ is true"; always and sometime: $\square A \equiv$ "it is always $A$ true", $\diamond A \equiv$ "it is sometime $A$ true"; knowledge and ignorance: $\square A \equiv$ "it is known that $A$ is true", $\diamond A \equiv$ "it is ignored $A$ is true".

The standard semantics of $\mathcal{L}_{\mathcal{M L}}$ formulas is the possible world semantics introduced by Kripke (see [15, 22] for many historical notes). A model for a set $T$ of $\mathcal{L}_{\mathcal{M} \mathcal{L}}$ formulas is a pair $\mathcal{M}=(\mathcal{F}, \mathcal{V})$, where $\mathcal{F}$ is a frame and $\mathcal{V}$ is an assignment for propositional variables $\mathcal{P}$ of $T$. More precisely, a frame is a pair $\mathcal{F}=(\mathcal{W}, \mathcal{R})$, where $\mathcal{W}$ is a non-empty set of objects, the possible worlds, and $\mathcal{R} \subseteq \mathcal{W} \times \mathcal{W}$ is a binary relation, the accessibility relation. An assignment $\mathcal{V}: \mathcal{P} \mapsto 2^{\mathcal{W}}$ is a mapping from propositional variables in $T$ to subsets of worlds in $\mathcal{W}$.

Definition 26 (a formula holds in a world $w$ ) Given an $\mathcal{L}_{\mathcal{M} \mathcal{L}}$ formula $A$ and a model $\mathcal{M}=(\mathcal{W}, \mathcal{R}, \mathcal{V})$, A holds in a world $w \in \mathcal{W}$, denoted as $\mathcal{M} \vDash_{w} A$, iff:

$$
\begin{array}{ll}
\mathcal{M} \models_{w} A, A \in \mathcal{P} \text { iff } w \in \mathcal{V}(A) & \mathcal{M} \models_{w} A \wedge B \text { iff } \mathcal{M} \models_{w} A \text { and } \mathcal{M} \models_{w} B \\
\mathcal{M} \models_{w} T & \mathcal{M} \models_{w} A \vee B \text { iff } \mathcal{M} \models_{w} A \text { or } \mathcal{M} \models_{w} B \\
\mathcal{M} \models_{w} \perp & \mathcal{M} \models_{w} A \rightarrow B \text { iff } \mathcal{M} \models_{w} A \text { implies } \mathcal{M} \models_{w} B \\
\mathcal{M} \models_{w} \neg A \text { iff } \mathcal{M} \models_{w} A & \mathcal{M} \models_{w} \square A \text { iff } \forall t \in \mathcal{W}, w \mathcal{R} \text { implies } \mathcal{M} \models_{w} A \\
\mathcal{M} \models_{w}(A) \text { iff } \mathcal{M} \models_{w} A & \mathcal{M} \models_{w} \diamond A \text { iff } \exists t \in \mathcal{W}, w \mathcal{R} \text { t and } \mathcal{M} \models_{w} A
\end{array}
$$

Definition $27\left(\mathcal{L}_{\mathcal{M} \mathcal{L}}\right.$ satisfiability) Given an $\mathcal{L}_{\mathcal{M} \mathcal{L}}$ formula $A$ and a model $\mathcal{M}=$ $(\mathcal{W}, \mathcal{R}, \mathcal{V}), A$ is satisfiable in $\mathcal{M}$, denoted as $\mathcal{M} \vDash A$, iff $\forall w \in \mathcal{W}, \mathcal{M} \vDash{ }_{w} A$

Definition 28 ( $\mathcal{L}_{\mathcal{M} \mathcal{C}}$ validity) Given an $\mathcal{L}_{\mathcal{M} \mathcal{L}}$ formula $A$ and a frame $\mathcal{F}, A$ is valid in $\mathcal{F}$, denoted as $\mathcal{F} \vDash A$, iff $\forall \mathcal{M}=(\mathcal{F}, \mathcal{V}), \mathcal{M} \models A$

Given a subset $\mathcal{T}_{\mathcal{A X}}$ of $\mathcal{L}_{\mathcal{M} \mathcal{L}}$ formulas, $\mathcal{T}_{\mathcal{A} \mathcal{X}}$ defines a modal logic if: (i) $\mathcal{T}_{\mathcal{A X}}$ includes all tautologies; (ii) $\mathcal{T}_{\mathcal{A X}}$ is closed under modus ponens and uniform substitution.

Definition 29 (Theorems) Given a logic $\mathcal{T}_{\mathcal{A X}}$ and an $\mathcal{L}_{\mathcal{M} \mathcal{L}}$ formula $A, A$ is a theorem of $\mathcal{T}_{\mathcal{A X}}$, denoted as $\vdash \tau_{\mathcal{A X}} A$, iff $A \in \mathcal{T}_{\mathcal{A X}}$.

Definition 30 (Deductibility) Given a logic $\mathcal{T}_{\mathcal{A X}}$ and a set $\Gamma \cup A$ of $\mathcal{L}_{\mathcal{M L}}$ formulas, 
$A$ is deducible from $\Gamma$ in $\mathcal{T}_{\mathcal{A X}}$, denoted as $\Gamma \vdash \tau_{\mathcal{A X}} A$, iff there exists $B_{1}, B_{2}, \ldots$, $B_{n} \in \Gamma$ such that: $\vdash \tau_{\mathcal{A X}} B_{1} \rightarrow\left(B_{2} \rightarrow\left(\ldots \rightarrow\left(B_{n} \rightarrow A\right) \ldots\right)\right)$

Definition 31 (Consistency) Given a logic $\mathcal{T}_{\mathcal{A X}}$ and an $\mathcal{L}_{\mathcal{M} \mathcal{L}}$ formula $A$ (a set of formulas $\Gamma), A(\Gamma)$ is consistent respect $\mathcal{T}_{\mathcal{A X}}$, denoted as $A(\Gamma) \forall \tau_{\mathcal{A X}} \perp$, iff is not true $\Gamma \vdash \tau_{\mathcal{A X}} \perp$.

Much of work in modal logic has concerned proof theory, soundness, completeness and decidability (see again $[22,11]$ ). From others two fundamental problems are distinguished for a given modal logic $\mathcal{T}_{\mathcal{A X}}$ and a class of frame $\mathcal{C}$ :

soundness $A \in \mathcal{T}_{\mathcal{A X}}$ implies $\forall \mathcal{F} \in \mathcal{C}, \mathcal{F} \vDash A$

completeness $\forall \mathcal{F} \in \mathcal{C}, \mathcal{F} \vDash A$ implies $A \in \mathcal{T}_{\mathcal{A X}}$

When for a set of frames $\mathcal{C}$ and for a logic $\mathcal{T}_{\mathcal{A X}}$, both soundness and completeness hold, it is said that $\mathcal{T}_{\mathcal{A X}}$ is determined by the set of frames $\mathcal{C}$.

A modal logic $\mathcal{T}_{\mathcal{A X}}$ can be syntacticly characterized using axioms or schemata. A schema is a collection of formulas included in $\mathcal{T}_{\mathcal{A X}}$ all having a common syntactic form. Describing a modal logic using specific axioms imposes specific constraints on the accessibility relation $\mathcal{R}$. The most studied modal logics are normal modal logics which are those that include the schema: $\mathrm{K}: \square(A \rightarrow B) \rightarrow(\square A \rightarrow \square B)$ and are close under the rule of necessitation: if $A \in \mathcal{T}_{\mathcal{A X}}$ then $\square A \in \mathcal{T}_{\mathcal{A X}}$ Each normal logic can be denoted using the notation: $K A X_{1} \ldots A X_{n}$, where each $A X_{i}$ denotes a specific axiom. In the Table 10 we report some well-known axiom.

\begin{tabular}{ccl}
$\begin{array}{c}\text { Name } \\
D\end{array}$ & $\begin{array}{c}\text { Axiom } \\
A \rightarrow \circ A\end{array}$ & Serial: $\forall w \exists t w \mathcal{R} t$ \\
\hline $\mathrm{T}$ & $\square A \rightarrow A$ & Reflexive: $\forall w w \mathcal{R} w$ \\
\hline 4 & $\square A \rightarrow \square \square A$ & Transitive: $\forall w \forall t \forall z w \mathcal{R} t \wedge t \mathcal{R} z \rightarrow w \mathcal{R} z$ \\
\hline $\mathrm{B}$ & $A \rightarrow \square \circ A$ & Symmetric: $\forall w \forall t w \mathcal{R} t \rightarrow t \mathcal{R} w$ \\
\hline 5 & $\circ A \rightarrow \square \circ A$ & Euclidean: $\forall w \forall t \forall z w \mathcal{R} t \wedge w \mathcal{R} z \rightarrow t \mathcal{R} z$
\end{tabular}

Table 10 The schemata for Modal Logics

The validity problem is decidable for most of modal logics, that is: for a given modal logic $\mathcal{T}_{\mathcal{A X}}$ there is an algorithm for determining for each modal formula $A$ whether or not $A \in \mathcal{T}_{\mathcal{A X}}$. In general, the computational complexity analysis of the validity problem for modal logics gives rise to intractable results, that is: the problem belongs to NP or expt-time classes [22].

For this work the main result follows from the coding of color markers into propositional modal formulas of a specific normal modal logic: i.e. $K 45 \equiv S 5$. $S 5$ is one well known modal logics called logic of necessity because it characterizes the notion of logical necessity. A logical necessary truth is one which is true in all possible worlds as for Green and Red data. Two important results hold for $S 5$ (see [22]):

1. S5 is determined by the class of recursive, symmetric and transitive frames

2. The validity problem for S5 is decidable and NP-complete. 


\section{APPENDIX 2 MULTI-VALUED LOGIC}

The language of $\mathcal{L}_{\mathcal{M} \mathcal{V}^{N}}$ is defined as for classical propositional logic. Given a set $\mathcal{P}$ of propositional variables, $\mathcal{L}_{\mathcal{M} \mathcal{V}^{N}}$ formulas are defined as follows:

Atoms: every $A \in \mathcal{P}$ and the two constants $\mathrm{T}, \perp$ are atomic propositional formulas of $\mathcal{L}_{\mathcal{M} \mathcal{V}^{N}}$

Formulas: if $A, B$ are formulas then: $\neg A, A \wedge B, A \vee B, A \rightarrow B,(A)$, are formulas of $\mathcal{L}_{\mathcal{M V}}$;

nothing else is a formula of $\mathcal{L}_{\mathcal{M} \mathcal{V}^{N}}$.

The semantics of a propositional multi-valued logic depends on the set of values chosen for defining the valuation function. Formally, a model for a set $T$ of $\mathcal{L}_{\mathcal{M} \mathcal{V}^{N}}$ formulas is a pair $\mathcal{M}=(\mathcal{F}, \mathcal{V})$ where $\mathcal{F}$ is a skeleton for logical connectives of $\mathcal{L}_{\mathcal{M} \mathcal{V}^{N}}$ and $\mathcal{V}: \mathcal{P} \mapsto \mathcal{N}$ is a valuation function that maps propositional variables onto $\mathcal{N}$. More precisely, $\mathcal{N}$ represents the domain of truth values and $\mathcal{A}=\{A(\wedge), A(\vee), A(\rightarrow), A(\neg)\}$ is a set of mapping on $\mathcal{N}$ of suitable arity associated with each logical connective* ${ }^{*}$ Given a model $\mathcal{M}=(\mathcal{N}, \mathcal{A}, \mathcal{V})$ an interpretation ()$^{\mathcal{V}}$ is defined as the homomorphic extension of $\mathcal{V}$ that maps $\mathcal{L}_{\mathcal{M} \mathcal{V}^{N}}$ formulas onto $\mathcal{N}$. That is, for any logical connective $o p\left(x_{1}, x_{2}, \ldots, x_{k}\right)$ of arity $\mathrm{k}$ it holds:

$\left(o p\left(x_{1}, x_{2}, \ldots, x_{k}\right)\right)^{\mathcal{V}}=A(o p)\left((x)_{1}^{\mathcal{V}},(x)_{2}^{\mathcal{V}}, \ldots,(x)_{k}^{\mathcal{V}}\right)$ and for any propositional variable $A \in \mathcal{P}$ it holds: $(A)^{\mathcal{V}} \equiv \mathcal{V}(A)$.

Definition $32\left(\mathcal{L}_{\mathcal{M} \mathcal{V}^{N}}\right.$ satisfiability) Given $F \in \mathcal{L}_{\mathcal{M V}^{N}}, U \subset \mathcal{N}$ and $\mathcal{M}=$ $(\mathcal{F}, \mathcal{V}),(F, U)$ is satisfiable in $\mathcal{M}$, denoted as $\mathcal{M} \models_{\mathcal{L}_{\mathcal{M} \mathcal{V}^{N}}}^{\mathcal{V}}(F, U)$, iff $(F)^{\mathcal{V}} \in U$.

Definition $33\left(\mathcal{L}_{\mathcal{M}^{N}}\right.$ validity) Given $F \in \mathcal{L}_{\mathcal{M V}^{N}}, U \subset \mathcal{N}$ and $\mathcal{F},(F, U)$ is valid in $\mathcal{F}$, denoted as $\mathcal{F} \models_{\mathcal{L}_{\mathcal{M} \mathcal{V}^{N}}}(F, U)$, iff $\forall \mathcal{M}=(\mathcal{F}, \mathcal{V}), \mathcal{M} \models_{\mathcal{L}_{\mathcal{M} \mathcal{V}^{N}}^{V}} F$.

Any valid formula is a tautology. Moreover, because for a given $\mathcal{L}_{\mathcal{M} \mathcal{V}^{N}}$ the skeleton $\mathcal{F}$ is also given, satisfiability and validity are usually related only to the valuation function $\mathcal{V}$ and not to the whole model $\mathcal{M}=(\mathcal{F}, \mathcal{V})$. In many cases between elements of $\mathcal{N}$ the true value is distinguished and therefor satisfiability and validity are considered with $U=\{$ true $\}$. Depending on $\mathcal{N}$ we have different multi-valued logics, for example if $\mathcal{N}=\{$ true, false $\}$ we have classical propositional logic. That is, propositional multi-valued logics generalize classical propositional logic. If $\mathcal{N}$ is finite and its cardinality is small (three or four) each $A(o p) \in \mathcal{A}$ is defined by a truth table, as we have done. Other possible multi-valued logics come from choosing $\mathcal{N}$ infinite or finite and unordered or ordered. In any case when $\mathcal{N}$ is a lattice $\Lambda=(N, \Pi, \sqcup)$ each logical connective is defined in terms of join $(\sqcup)$ and meet $(\Pi)$ operations over the lattice elements $\mathcal{N}$ (see [13] for further details).

\footnotetext{
"More in general, a multi-valued logic $\mathcal{L}_{\mathcal{M} \mathcal{V}^{N}}$ can extend the standard set of logical connectives with new ones, providing for them specific maps $A(o p): \mathcal{L}_{\mathcal{M} \mathcal{V}^{N}} \mapsto \mathcal{N}$.
} 


\section{APPENDIX 3 TABLES}

\begin{tabular}{|c|c|}
\hline SSN & TELEPHONE \\
\hline $245569789: \diamond \mathbf{R}$ & $703-708-4429: \square$ R \\
\hline 245569787:0 R & 703-993-1628: $\bullet \mathbf{R}$ \\
\hline \multicolumn{2}{|c|}{$\tau_{(\star, \ddagger)}^{c}$} \\
\hline SSN & TELEPHONE \\
\hline 234599133: व G & $703-993-1629: \square \mathrm{G}$ \\
\hline 324599587:0 G & $703-708-4429: \square \mathrm{G}$ \\
\hline
\end{tabular}

\begin{tabular}{|c|c|}
\hline SSN & TELEPHONE \\
\hline 325599585:०G & 703-993-1628: $\mathbf{R}$ \\
\hline 324499581:० R & $703-708-4427: \circ \mathrm{G}$ \\
\hline 245569789:०G & 703-993-1629: $\bullet \mathbf{R}$ \\
\hline 245569789:०R & 703-708-4429: $\square \mathbf{R}$ \\
\hline 245569787: $\mathbf{R}$ & 703-993-1628:० R \\
\hline \multicolumn{2}{|c|}{$\mathcal{T}_{(\star, \dagger)}^{c}$} \\
\hline SSN & TELEPHONE \\
\hline 234599133:口 G & $703-993-1629: 0 \mathrm{G}$ \\
\hline $324599587: 0 \mathrm{G}$ & $703-708-4429: 0 \mathrm{G}$ \\
\hline 324499581:० R & $703-708-4427: \circ G$ \\
\hline $245569789: \circ G$ & 703-993-1629:० R \\
\hline
\end{tabular}

Table 11 The projection of a marked relation

\begin{tabular}{|c|c|c|}
\hline SSN & BILL & LAST-BILL \\
\hline $324499581: \circ G$ & $1503: 0 \mathrm{G}$ & $1803: \square \mathrm{G}$ \\
\hline 325599585: $\square \mathrm{G}$ & $1508: 0 \mathrm{G}$ & $2008: 0 \mathrm{G}$ \\
\hline \multicolumn{3}{|c|}{$\tau_{(\sim, \ddagger)}^{c}$} \\
\hline ssN & BILL & LAST-BILL \\
\hline $325599585: 0 \mathbf{R}$ & 150s:०R & 200s:० R \\
\hline $245569789: 0 \mathbf{R}$ & $3008: 0$ R & $4008: \square R$ \\
\hline
\end{tabular}

\begin{tabular}{|c|c|c|}
\hline \multicolumn{3}{|c|}{$\mathcal{T}_{(\sim, \dagger)}^{c}$} \\
\hline SSN & BILL & LAST-BILL \\
\hline $324499581: \circ \mathrm{G}$ & $1508: 0 \mathrm{G}$ & $1808: 0 \mathrm{G}$ \\
\hline 325599585:口G & 150s:0 G & 2008:०G \\
\hline 325599585:० R & $1508: 0 \mathbf{R}$ & 2008:० R \\
\hline 245569789:०R & $1508: \circ G$ & $1008: 0 \mathbf{R}$ \\
\hline \multicolumn{3}{|c|}{$\tau_{(\star, \dagger)}^{c}$} \\
\hline SSN & BILL & LAST-BILL \\
\hline $325599585: \square \mathbf{R}$ & 2508:० G & $3008: \circ \mathrm{G}$ \\
\hline 325599585: $\square \mathbf{R}$ & 250\$:० R & $1508: \circ G$ \\
\hline 325599585: ० R & $150 s: \circ \mathbf{R}$ & 200s:० R \\
\hline 245569789:०R & $1508: 0 \mathrm{G}$ & $1008: 0 \mathbf{R}$ \\
\hline 245569789:॰R & 300\$:0 R & 4008: $\square \mathbf{R}$ \\
\hline
\end{tabular}

Table 12 The result of $\sigma_{\mathcal{F}}^{c}$ where $\mathcal{F}=$ BILL $>$ LAST-BILL 


\begin{tabular}{|c|c|c|}
\hline \multicolumn{3}{|c|}{ VIEW } \\
\hline SSN & BILL & LAST-BILL \\
\hline 234599133:口G & 200s:םG & 250s:० R \\
\hline 324499581:०G & $1508: 0 \mathrm{G}$ & $180 \$: 0 G$ \\
\hline 324499581: $\mathbf{R}$ & 2008:口 G & 400s:口 R \\
\hline 324499581: $\mathbf{R}$ & 400\$:० G & 100s: : R \\
\hline $324499581: 0 \mathrm{G}$ & $3008: 0 \mathbf{R}$ & 4008: $\square \mathrm{R}$ \\
\hline 325599585: Q R & 2508:० G & 300s:० G \\
\hline 325599585:口 G & 1508:0 G & 200s:० G \\
\hline 325599585: R R & 2508:० R & $1508: \circ G$ \\
\hline 325599585:० R & $1508: \circ \mathbf{R}$ & 200s:० R \\
\hline 245569789:० R & $1508: \circ \mathbf{G}$ & $1008: 0 \mathbf{R}$ \\
\hline 245569789:०R & 3008:0 R & 400s: : R \\
\hline
\end{tabular}

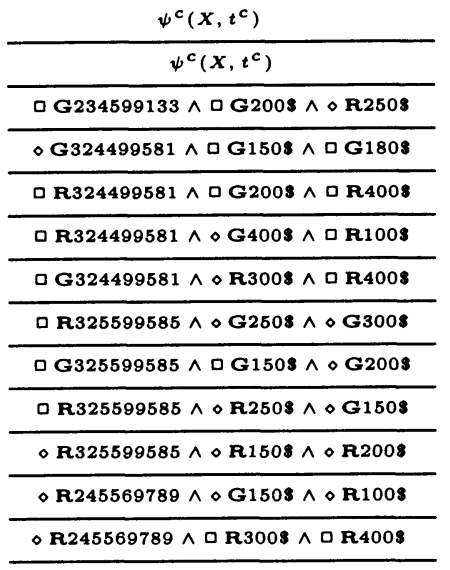

Table 13 Relation view and the corresponding tuple constraints

\begin{tabular}{|c|c|}
\hline $\mathcal{T}_{(\star, I)}^{c}\left(t^{c}, X, X\right)$ & $\mathcal{T}_{(\sim, I)}^{c}\left(t^{c}, X, X\right)$ \\
\hline 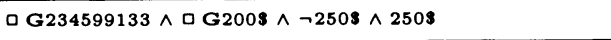 & $\perp \wedge \perp \wedge \neg 250 s$ \\
\hline $324499581 \wedge \square G 150 s \wedge \square G 180 s$ & $\neg 324499581 \wedge 324499581 \wedge \perp \wedge \perp$ \\
\hline$\perp \wedge 0$ G200s $\wedge \perp$ & 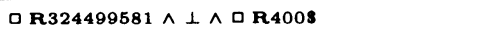 \\
\hline$\perp \wedge 400 \Omega \perp$ & 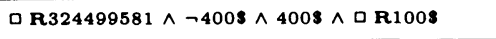 \\
\hline$\square$ G324499581 $\neg \neg 3003 \wedge 300 \wedge \wedge$ & $\perp \wedge \neg 300 s \wedge \square \mathbf{R 4 0 0 s}$ \\
\hline$\perp \wedge 250 s \wedge 300 s$ & 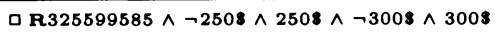 \\
\hline$\triangle G 325599585 \wedge \square G 1508 \wedge 2008$ & $\perp \wedge \perp \wedge \neg 2008 \wedge 2008$ \\
\hline$\perp \wedge \neg 2508 \wedge 2508 \wedge 1508$ & 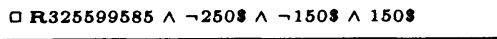 \\
\hline$\neg 325599585 \wedge 325599585 \wedge \neg 1503 \wedge 150 \diamond \wedge \neg 200 \diamond \wedge 2003$ & $\neg 325599585 \wedge \neg 1508 \wedge \neg 2008$ \\
\hline$-245569789 \wedge 245569789 \wedge 1508 \wedge \neg 100 s \wedge 1008$ & $\neg 245569789 \wedge \neg 1508 \wedge 1508 \wedge \neg 1008$ \\
\hline$\neg 245569789 \wedge 245569789 \wedge \perp \wedge \perp$ & 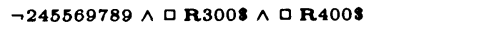 \\
\hline
\end{tabular}

Table 14 The query constraints for relation view

\begin{tabular}{ccl}
\hline SSN & TELEPHONE & COMPANY \\
\hline 234599133:0 R & 703-993-1629:0 G & Sprint:0 G \\
\hline 234599133: 0 R & 703-993-1629:0 G & Sprint:0 G \\
\hline
\end{tabular}

Table 15 The result of pre- $\aleph_{=}^{c}$ under $\ddagger$ interpretation 


\begin{tabular}{|c|c|c|}
\hline SSN & TELEPHONE & COMPANY \\
\hline 234599133:० G & 703-708-4428:०R & AT\& $T: 0 R$ \\
\hline 234599133:0 R & $703-993-1629: \circ \mathrm{G}$ & AT\& $\mathbf{T}: \square \mathrm{G}$ \\
\hline 234599133:口 R & $703-993-1629: \circ$ G & Sprint: 0 G \\
\hline 245569789:0 G & $703-993-1628: \circ \mathrm{G}$ & AT\& $T: \square$ G \\
\hline 234599133:० G & $703-708-4427: \circ \mathrm{G}$ & AT\& $T: 0 R$ \\
\hline 234599133:0 R & 703-708-4429:०R & $A T \& T: \square G$ \\
\hline 234599133:口 R & $703-993-1629: \square \mathrm{G}$ & Sprint: $\mathbf{G}$ \\
\hline 245569789:0 G & $703-708-4429:$ & AT\& $\mathbf{T}: \mathbf{Q G}$ \\
\hline
\end{tabular}

Table 16 The result of pre- $\bowtie_{=}^{c}$ under $\dagger$ interpretation

\begin{tabular}{ccc}
\hline SSN & TELEPHONE & COMPANY \\
\hline 0 & 0 & $\emptyset$ \\
\hline
\end{tabular}

Table 17 The result of $\aleph_{=}^{c}$ under $\ddagger$ interpretation

\begin{tabular}{ccl}
\hline SSN & TELEPHONE & COMPANY \\
\hline 234599133:० G & $703-708-4428: 0$ R & AT\&T:०R \\
\hline 245569789: O G & $703-993-1628: 0$ G & AT\&T:DG \\
\hline 234599133:० G & $703-708-4427: 0$ G & AT\& $\&: \circ R$ \\
\hline 245569789:0 G & $703-708-4429: 0$ R & AT\&T:DG \\
\hline
\end{tabular}

Table 18 The result of $\bowtie_{=}^{c}$ under $\dagger$ interpretation

\begin{tabular}{|c|c|c|c|}
\hline SSN & TELEPHONE & PAYMENT & COMPANY \\
\hline 234599133:० R & $703-708-4429: 0 \mathrm{G}$ & 703-993-1629:口 G & Sprint: 0 G \\
\hline 234599133: व R & $703-993-1629: \circ G$ & 703-993-1629:םG & Sprint: $\square$ G \\
\hline 245569789:0 G & $703-993-1628: \circ \mathrm{G}$ & $703-993-1629: 0 \mathrm{G}$ & Sprint: $\mathbf{Q G}$ \\
\hline
\end{tabular}

Table 19 The result of pre- $\bowtie_{\Theta}^{c}$ under $\ddagger$ interpretation 


\begin{tabular}{|c|c|c|c|}
\hline SSN & TELEPHONE & PAYMENT & COMPANY \\
\hline 234599133:० R & $703-708-4429: 0 \mathrm{G}$ & $703-993-1629: 0 \mathrm{G}$ & Sprint: $\square$ G \\
\hline 234599133:० G & 703-708-4428: $\bullet \mathbf{R}$ & 703-708-4427: & AT\& $\&$ : $० \mathbf{R}$ \\
\hline 234599133:०G & 703-708-4428: $\mathbf{R}$ & 703-708-4429:॰ R & AT\&T: $\mathbf{T} \mathbf{G}$ \\
\hline 234599133:ם R & $703-993-1629: \circ \mathrm{G}$ & 703-708-4429:० R & AT\&T:DG \\
\hline 234599133:口 R & $703-993-1629: \circ \mathrm{G}$ & $703-993-1629: 0 \mathrm{G}$ & Sprint: $\square \mathbf{G}$ \\
\hline 245569789:0 G & $703-993-1628: \circ G$ & 703-708-4429:० R & AT\&T:口G \\
\hline 245569789:口G & $703-993-1628: \diamond \mathrm{G}$ & 703-993-1629:口G & Sprint: $\square$ G \\
\hline
\end{tabular}

Table 20 The result of pre- $\bowtie_{\Theta}^{c}$ under $\nmid$ interpretation

\begin{tabular}{cccl}
\hline SSN & TELEPHONE & PAYMENT & COMPANY \\
\hline 245569789:0 G & $703-993-1628: 0 \mathrm{G}$ & $703-993-1629: 0 \mathrm{G}$ & Sprint: $\mathrm{G}$ \\
\hline
\end{tabular}

Table 21 The result of $\bowtie_{\Theta}^{c}$ under $\ddagger$ interpretation

\begin{tabular}{|c|c|c|c|}
\hline SSN & TELEPHONE & PAYMENT & COMPANY \\
\hline 234599133:0 R & 703-708-4429:0 G & 703-993-1629:口G & Sprint: 0 G \\
\hline 234599133:० G & 703-708-4428:०R & $703-708-4427: 0 \mathrm{G}$ & AT\& $T: 0 R$ \\
\hline $234599133:$ : G & 703-708-4428: $\mathbf{R}$ & 703-708-4429:० R & AT\&T:DG \\
\hline 245569789:0 G & $703-993-1628: \circ \mathrm{G}$ & 703-708-4429:०R & AT \& $\mathbf{T}: \square \mathrm{G}$ \\
\hline 245569789:0 G & $703-993-1628: \circ \mathrm{G}$ & $703-993-1629: \square \mathrm{G}$ & Sprint: $\mathbf{Q G}$ \\
\hline
\end{tabular}

Table 22 The result of $\bowtie_{\Theta}^{c}$ under $\dagger$ interpretation 